\title{
HECTOR, a fast simulator for the transport of particles in beamlines
}

\author{
J. de Favereau, X. Rouby and K. Piotrzkowski * \\ Center for Particle Physics and Phenomenology (CP3), Université catholique de Louvain \\ B-1348 Louvain-la-Neuve, Belgium
}

November 2, 2018

\begin{abstract}
Computing the trajectories of particles in generic beamlines is an important ingredient of experimental particle physics, in particular regarding near-beam detectors. A new tool, HЕстоR, has been built for such calculations, using the transfer matrix approach and energy corrections. The limiting aperture effects are also taken into account. As an illustration, the tool was used to simulate the LHC beamlines, in particular around the high luminosity interaction points (IPs), and validated with results of the MAD-X simulator. The LHC beam profiles, trajectories and beta functions are presented. Assuming certain forward proton detector scenarios around the IP5, acceptance plots, irradiation doses and chromaticity grids are produced. Furthermore, the reconstruction of proton kinematic variables at the IP (energy and angle) is studied as well as the impact of the misalignment of beamline elements.
\end{abstract}

PACS: 29.27.-a ; 41.85.Ja

Keywords: Particle transport, transfer matrix, LHC beamline, forward detectors, misalignment

Preprint: CP3-07-13

\section{Introduction}

In the context of the LHC beamlines and in particular of very forward, near beam detectors, we introduce a new fast simulator, called ${ }^{1,2}$ HECTOR, for the transport of single particles through generic beamlines, although primarily dedicated to the LHC. The simulator is based on a linear approach of the beamline optics, implementing transport matrices from the optical element magnetic effective length, and with correction factors on magnetic strength for particles with non nominal energy. HECTOR deals with the computation of the position and angle of beam particles, and the limiting aperture of the optical elements. It has been designed to be fast, light and user friendly and its object oriented structure, using the RoOT framework [1], helps its usage, its maintenance and the future improvements.

In forward physics, the measurement of the position and the angle of particles in dedicated detectors (as so-called roman pots for example) hundreds of meters away from the interaction point (IP), helps in

\footnotetext{
*Email addresses: jdf@fynu.ucl.ac.be and rouby@fynu.ucl.ac.be and krzysztof.piotrzkowski@cern.ch

${ }^{1}$ The user guide is available as an appendix of this document.

${ }^{2}$ An homonymic program is used in the DIS community, for the calculation of QED, QCD and electroweak corrections to e p and lepton+- N deep inelastic neutral and charged current scattering.
} 
reconstructing the kinematics of the event at the related central detector. HECTOR links the information from very forward detectors (VFDs) to the one from the central detector, by precise calculation of the particle trajectory.

We present here the physics content and the simulation techniques used in HECTOR, as well as its overall performance. The validation of HECTOR output comes from direct comparisons with the MAD-X simulator [2], for the beta functions and relative positions of both LHC beams. Some physics results, like beam profiles and irradiation levels due to the forward proton from inelastic pp $\rightarrow \mathrm{pX}$ interactions are plotted for two reasonable scenarios for the very forward detectors. Finally, first studies of the forwardscattered proton kinematics reconstruction are performed, resulting in the discussion of limiting factors and best strategies for the VFD designs.

\section{The LHC beamlines}

Even if HeCTOR's class structure is compatible with the most frequent beamline elements, the physics motivation on backstage is driven by the forward physics at the LHC. This includes detection of protons which have for example been quasi-elastically scattered by exchange of a photon. The reconstruction of the proton momentum using information from a VFD requires a good knowledge of the LHC beams and related beamlines. Such a beamline is described here below ; note that both LHC beams are basically identical.

\subsection{Beamline elements and beam parameters}

The position and lateral size of the LHC beams are mainly controlled by two types of elements :

- focusing (quadrupoles) or deflecting (dipoles, kickers) magnets;

- collimators and optical element apertures, limiting the lateral beam extension.

Quadrupole magnets can focus the beam either vertically or horizontally. Dipole magnets also come in two kinds : rectangular dipoles with a straight shape, and sector dipoles which are bent to match the beam curved trajectory. The former are mainly used in the long and short straight sections (LSS, SSS) of the beamline, while the latter are placed in the dispersion suppressors (DSs) and the bending sections (ARCs) [3], [4]. The kickers are dipole magnets dedicated to produce the crossing angle at the IP.

Some parameters are of direct interest when describing the nominal $7 \mathrm{TeV}$ proton beams:

- the horizontal and vertical emittances $\epsilon_{x, y} \sim 500 \times 10^{-6} \mu \mathrm{m}$, which are constant along the orbit according to Liouville's theorem ;

- the lateral beam size at the IP $\sigma=16.6 \mu \mathrm{m}=\sqrt{\epsilon \beta^{*}}$, where $\beta^{*}$ is value $(=0.55 \mathrm{~m})$ at the IP of the so-called beta-function ;

- the lateral vertex size (or the transverse size of collision region) $\sigma^{v}=11.76 \mu \mathrm{m}=\sigma / \sqrt{2}$;

- the angular divergence at the IP $\sigma_{\theta}=30.2 \mu \mathrm{rad}=\sqrt{\epsilon / \beta^{*}}$;

- the crossing angle is equal to $2 \times 142.5 \mu \mathrm{rad}$, in vertical (horizontal) plane at the IP1 (IP5) ;

- the energy spread $\sigma_{E}=1.129 \times 10^{-4} E$. 


\subsection{Beamline map}

From here onwards, focus will be put on interaction region 5 (IR5). The elements affecting the beam will be described starting at interaction point 5 (IP5), going eastward when looking from the center. This is the propagation direction of beam 1 . The other side of the beamline is very similar. As the region of interest for the forward physics related detectors extends up to approximately $500 \mathrm{~m}$ from the IP, our description of the beamline stops there. Within this part of the beamline, all aperture shapes are rectellipses [4].

\section{Before $85 \mathrm{~m}$ from IP5}

After the IP, beam 1 gets separated from the incoming beam 2 because of the crossing angle. The two beam paths stay close $(\sim 4 \mathrm{~mm})$ for the first $85 \mathrm{~m}$. They encounter the first three collimators at $19 \mathrm{~m}$, $45 \mathrm{~m}$ and $55 \mathrm{~m}$ from the IP (the so-called low- $\beta$ inner triplet), then a kicker $(30 \mathrm{~m})$ and then quadrupoles (H: 23 and $47 \mathrm{~m}$; V: 32 and $39 \mathrm{~m}$ ) and consecutive dipoles (R-Bend) from 60 to $81 \mathrm{~m}$, which deviate the two beam paths to lead them to their final separation of $194 \mathrm{~mm}$. The dipoles are positioned symmetrically with respect to the two beams.

\section{From $85 \mathrm{~m}$ to $269 \mathrm{~m}$ from IP5}

At about $160 \mathrm{~m}$ from the IP big R-Bend dipoles are used to make the beam orbits parallel, so from there on the beams get to their nominal relative positions. These dipoles are positioned in such a way that the beam 1 (2) enters (exits) it perpendicularly. Four more collimators are located respectively at 141, 149, 184 and $256 \mathrm{~m}$ from the IP5. Two kickers, at 165 and $199 \mathrm{~m}$, lead them to the ideal path that they would have followed if there were not any crossing angle, due to the so-called closed bump orbit. The beam is kept under control with five more quadrupoles (H: 194, 260, 264 ; V: 168 and $226 \mathrm{~m}$ ) and one dipole $(153 \mathrm{~m})$. This is the end of the straight section.

\section{From 269 m onwards}

There the beam path starts its bending. The beamline is composed in alternations of quadrupoles $(>6)$ to keep the beam focused and sector dipoles ( $>8$ S-dipoles) to bend it. The first sextupoles with non-zero field occur only after $439 \mathrm{~m}$ from the IP.

\section{Simulation techniques}

\subsection{Physical description}

The simulator relies on a linear approach to single particle propagation. A beamline consists of a set of optical elements, amongst dipoles, quadrupoles, drifts, collimators, kickers and VFDs. The optical elements are described by their magnetic field, their length and their aperture. In turn, a set of particles, with all possible smearings of initial positions, angles or energies, is propagated by HECTOR through the beamline, particle by particle. In electromagnetism, the influence of an external magnetic field is given by the Lorentz force. Let's consider the Taylor expansion of the vertical component of magnetic field $B_{y}$, around its central value :

$$
\frac{e}{p} B_{y}(x)=\frac{e}{p} B_{y}+\frac{e}{p} \frac{\partial B_{y}}{\partial x} x+\frac{1}{2} \frac{e}{p} \frac{\partial^{2} B_{y}}{\partial x^{2}} x^{2}+\ldots
$$

where $p$ is the momentum of the particle and $e$ its electric charge. The terms of this sum are interpreted as respectively dipolar $\left(\frac{1}{R}=\frac{e}{p} B_{y}\right)$, quadrupolar $\left(k=\frac{e}{p} \frac{\partial B_{y}}{\partial x}\right)$ and sextupolar fields. In the co-moving coordinate system, neglecting small deviations $(x \ll R, y \ll R)$ and small momentum loss $(\Delta p \ll p)$, this leads to the following equation of motions for a particle traveling along path length $s$ through a magnetic element [5] : 


$$
\left\{\begin{array}{l}
x^{\prime \prime}(s)+\left(\frac{1}{R^{2}(s)}-k(s)\right) x(s)=\frac{1}{R(s)} \frac{\Delta p}{p} \\
y^{\prime \prime}(s)+k(s) y(s)=0 .
\end{array}\right.
$$

The solution $\left(x(s), x^{\prime}(s), y(s), y^{\prime}(s)\right)$ to these equations can be expressed as a linear combination of the initial values $\left(x_{0}, x_{0}^{\prime}, y_{0}, y_{0}^{\prime}\right)$, where the rotation matrices are defined by the properties of the optical element (length and magnetic field). The typical values $R \sim 200 \mathrm{~m}$ and $x<0.01 \mathrm{~m}$ match the first assumption $(x \ll R)$. As $\Delta p \ll p$ is not always verified, we apply a further correction to the magnetic field (for mode details, see section 3.2) of each optical element of the beamline.

In other words, for the simulation of the transport of a particle in a beamline, each beam particle is represented by a phase space vector and each optical element by a transfer matrix by which the vector is multiplied. The propagation of a single particle is thus the rotation of the phase space vector by the $n$ optical element matrices.

$$
X(s)=X(0) \underbrace{M_{1} M_{2} \ldots M_{n}}_{M_{\text {beamline }}}
$$

The whole beamline is modeled as a single transport matrix acting on each particle phase space vector (no intrabeam interactions). The optical element description also refers to its physical aperture. When a particle is propagated through an optical element, two tests check whether its path is compatible with the element acceptance or not, at its entrance and exit. The fact that a bent path could hit a central part of the element is neglected. A particle which does not pass through the element acceptance is flagged, allowing to know the stopping element for given particle parameters (for the computation of acceptance and irradiation rates for instance).

\subsection{Implementation}

The simulator has an object-oriented design, using the $\mathrm{C}++$ Rоoт framework [1], with dedicated classes describing :

- the beam particles. The 6 -components phase space vector is $X=\left(x, x^{\prime}, y, y^{\prime}, E, 1\right)$, with horizontal $\left(x, x^{\prime}\right)$ and vertical $\left(y, y^{\prime}\right)$ coordinates and angles ; $E$ is the particle energy. The sixth component is just a factor used to add an angular kick on the particle momentum direction.

- the optical elements (dipoles, drifts...) inheriting from a common class.

The $6 \times 6$ transport matrices can be decomposed into blocks

$$
\mathbf{M}_{\text {units }}=\left(\begin{array}{cccccc}
\mathcal{A} & \mathcal{A} & 0 & 0 & 0 & 0 \\
\mathcal{A} & \mathcal{A} & 0 & 0 & 0 & 0 \\
0 & 0 & \mathcal{B} & \mathcal{B} & 0 & 0 \\
0 & 0 & \mathcal{B} & \mathcal{B} & 0 & 0 \\
\mathcal{D} & \mathcal{D} & 0 & 0 & 1 & 0 \\
K & K & K & K & 0 & 1
\end{array}\right)
$$

where

- $\mathcal{A}$ (and $\mathcal{B}$ ) blocks refer to the action (focusing, defocusing, drift) on horizontal (and vertical, resp.) coordinate and angle.

- $\mathcal{D}$ terms reflect the dispersion effects of the horizontal dipoles on off-momentum particles.

- $K$ factors are the angular action of kickers. 
A beamline is the implementation of a list of optical elements with such transport matrices. The introduction of dispersion terms allow a proper description of off-momentum particles $(\Delta p / p \neq 0)$. The dispersion function can be defined from eq. (1), for horizontal dipoles $(k=0)$, taking $\Delta p / p=1$ :

$$
D^{\prime \prime}(s)+\frac{1}{R^{2}} D(s)=\frac{1}{R}
$$

Developing the solution of this equation leads to a correction term for the deflection of off-momentum particles in the dipoles :

$$
x_{\text {off-momentum }}(s)=x(s)+D(s) \frac{\Delta p}{p} .
$$

For explicit description of these $\mathcal{D}$ terms, refer to the appendix, Eq. (9). Besides the dispersion correction which is valid for low $\Delta p$, the actual dependence of the particle deflection on its momentum is also taken into account. This is the chromaticity, or the energy dependence of the transport matrix, implemented by rescaling the magnetic field terms $(R, k, K)$ with a factor $\left(\frac{p}{p-\Delta p}\right)$. The propagation of particles different from protons is also possible by rescaling these magnetic field terms :

$$
k_{i}\left(\Delta E, m_{p}, q_{p}\right)=k_{i} \frac{p_{0}}{p_{0}-\Delta p} \frac{q_{p}}{q_{\text {proton }}} \quad, \quad k_{i}=R, k, K
$$

where $q_{p}$ and $m_{p}$ are the particle charge and mass, respectively. The Root framework easily interfaces HeCTOR with the output of the high energy physics event generator PYTHIA [8]. Forward particles from the final state can be then propagated through the beamline via HECTOR, for example in photon induced interactions.

As Hector has been designed for the LHC beamlines, one class can parse the official LHC optics tables, interfacing them directly into the code at run-time. For a better compatibility with different table layout, Hector can recognize column headers and is thus compliant with different table types [9]. All the default parameters are hard coded (in H_Parameters.h), like nominal beam energy, position and angle, and their divergences, as well as the nominal crossing angle, but these default value can obviously be rewritten at run time. The effects of the kickers or the bending of the sector magnets can also be easily switched on or off. For the following analysis, LHC optics version 6.500 has been used, corresponding to $\beta^{*}=0.55 \mathrm{~m}$ at the IP1 and IP5.

Limitations : sextupoles and magnets of higher order (multipoles) are neglected in HECTOR (harmless the first $430 \mathrm{~m}$ after the IP). The energy dependence of the transfer matrices is taken into account, but neither beam-beam interactions, nor the field non-uniformities. The beam particle mass is also neglected compared to its energy.

\section{Validation and performances}

\subsection{Performances}

Hector has been designed to be fast and light. All the code and its library altogether weight less than $2.5 \mathrm{MB}$ for about 12000 lines of code. The figure 1 depicts its performance, leading for a first estimate to a CPU time of $4 \mu \mathrm{s}$ per beam particle per beamline optical element at the LHC. As expected, the CPU time is linear with the number of optical elements and the number of protons.

\subsection{Validating Hector with Mad-X}

In order to validate HECTOR, cross-checks have been made with the output of MAD-X [2], such as the beam beta functions (Fig. 2) and the beam relative positions (Fig. 3). For each point, the beta functions are computed from the beam emittance and the RMS of its profile in the transverse plane. The beam beta 
Computing time for 10000 particles

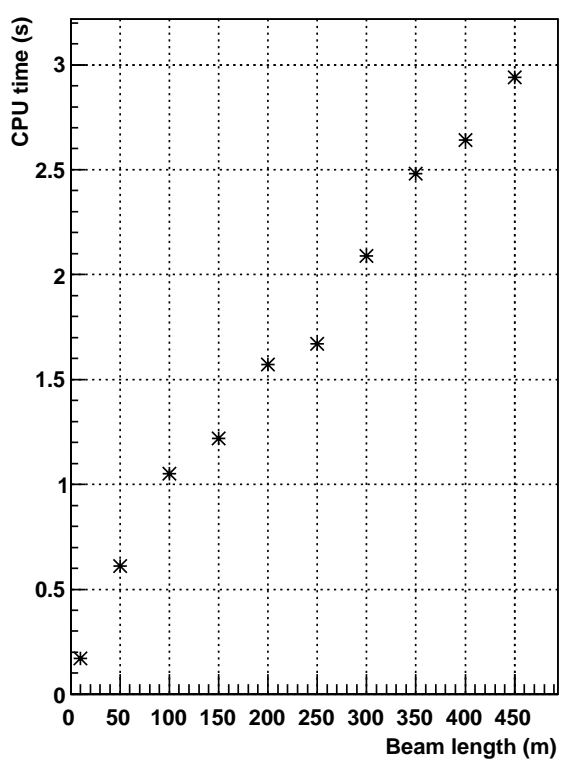

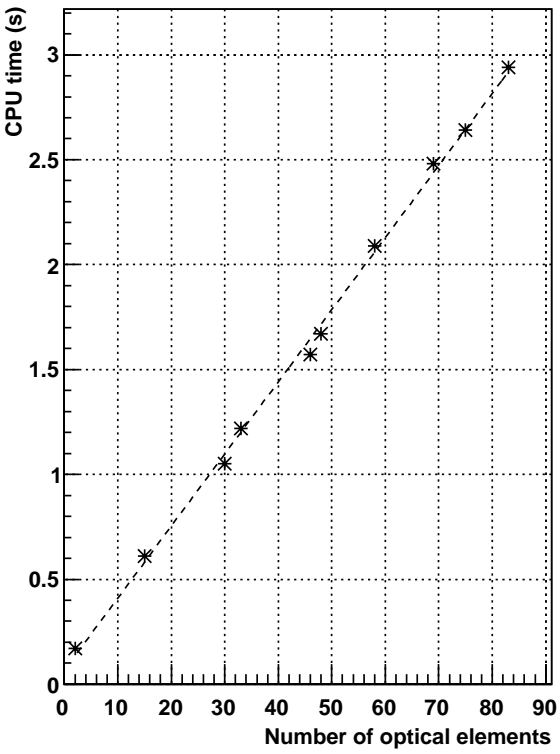

Figure 1: HECTOR speed performance: CPU time with respect to the propagation distance (left) and the number of optical elements (right) in the beamline, computed for a $10^{4}$ particle beam around the IR5. As the propagation of particles corresponds to the multiplication of matrices, the computation time is directly proportional to the amount of optical elements in the beamline, as well as the number of beam particles. For a time estimation for large number of particles and long beamlines, a good approximation is $4 \mu s$ per optical element (including drifts) per particle. For this test (3.43 $\mu$ s per element per particle), Hector is run on a regular laptop with $1.7 \mathrm{GHz}$ Intel Pentium Centrino processor, Linux rel. Ubuntu.

functions, which describe the lateral beam size variation along the orbit, as output by HECTOR, match perfectly the results from MAD-X. The lateral beam position shown in Fig. 3 is relative to its ideal path, defined for disabled kickers (and no crossing angle). These graphs (Fig. 2 and 3), and their equivalents in backward direction, are the strongest tests for HECTOR, where smearings of position, angle and energy are applied as previously explained.

\subsection{Validating off-beam energy simulation}

The comparisons of HECTOR and MAD-X results directly validate only the simulation of a 7 TeV proton propagation, and additional checks are necessary to validate simulations of the off-beam energy particles. This has been done by comparing numerically the exact calculations and the HECTOR predictions for a couple of proton trajectories at energies between 10 and $100 \%$ of the beam energy. It was found that for all the energies of interest for the VFDs, i.e. for energies above $80 \%$ of the beam energy, HECTOR remains very accurate, providing the particle lateral positions with precision at least at a few micron level. 


\section{$\beta$ functions - beam 1, forward}

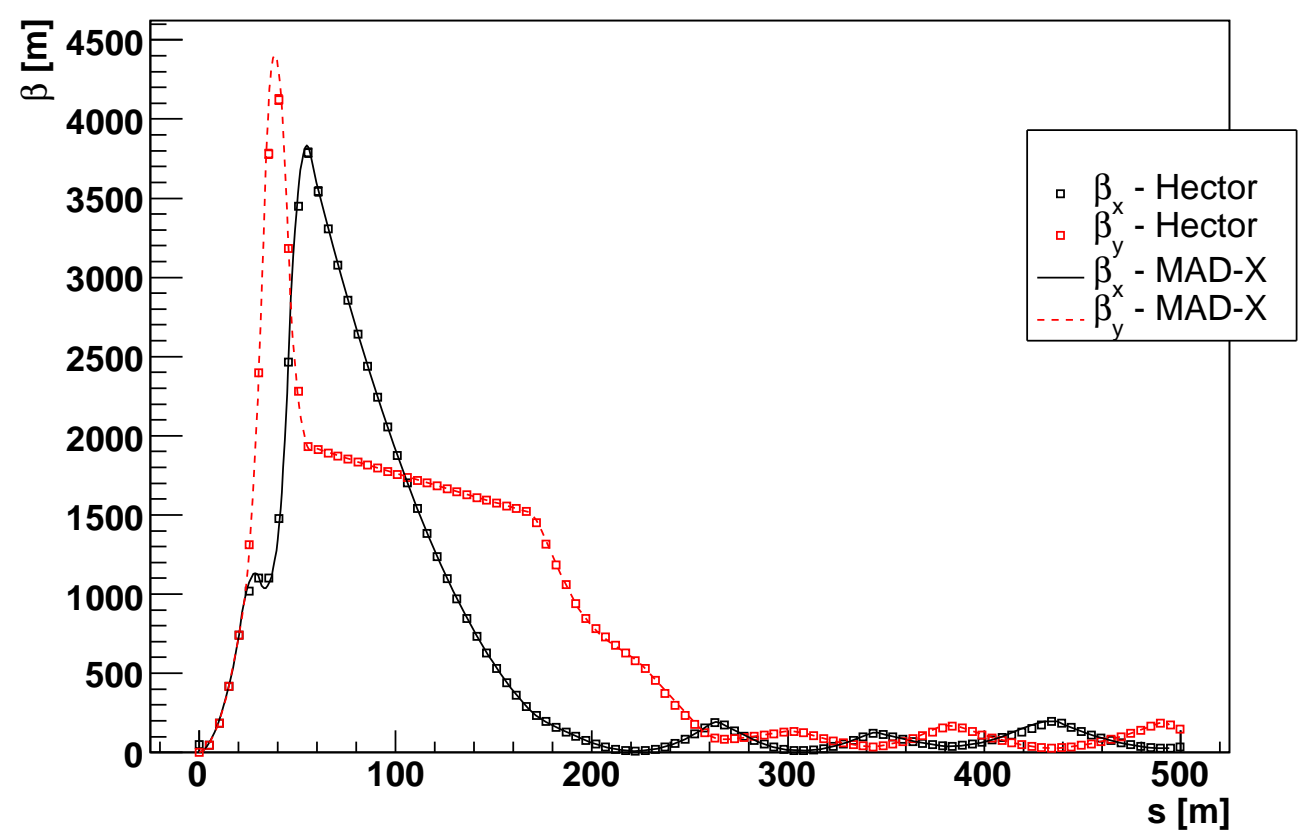

\section{$\beta$ functions - beam 2, forward}

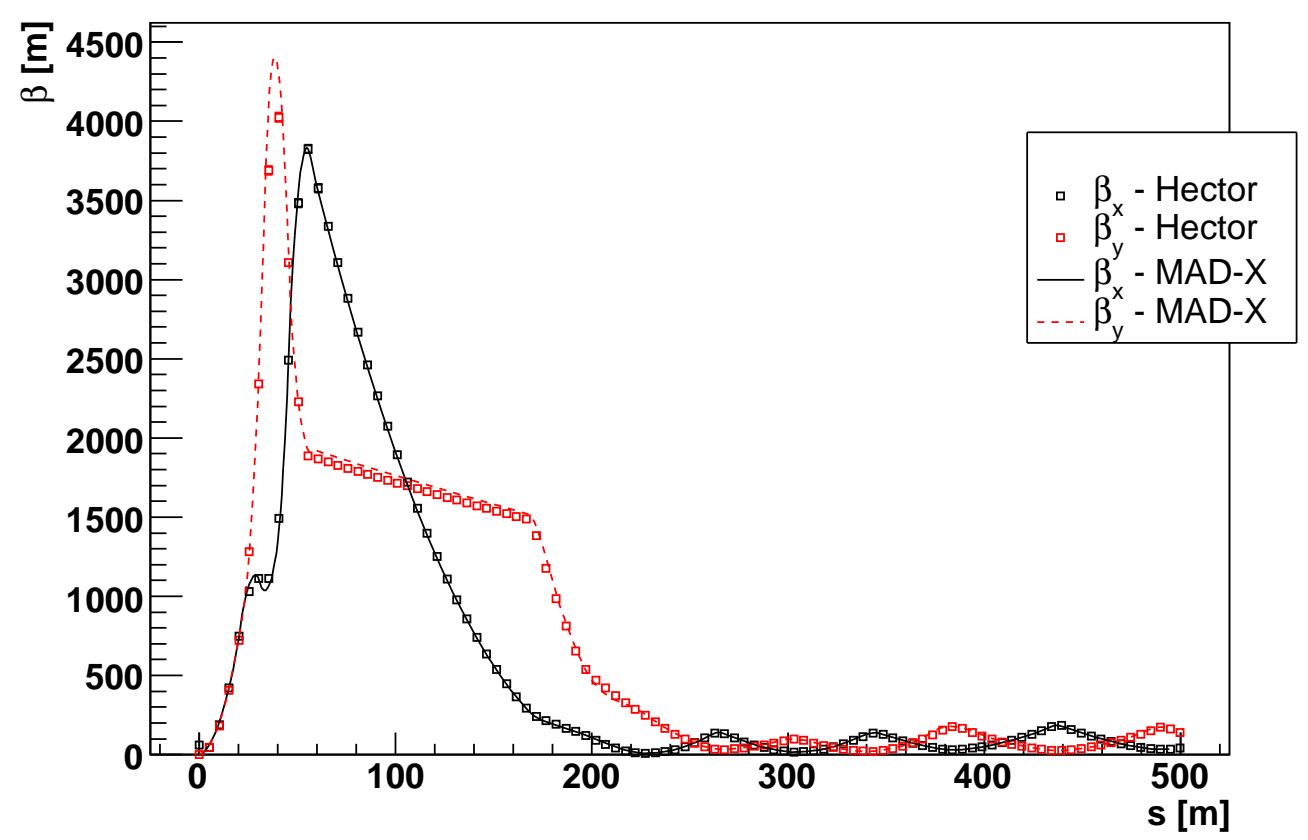

Figure 2: Beta functions $\beta_{x}$ and $\beta_{y}$ for beams 1 (above) and 2 (below) near the IP5, going forward: these functions depict the beam size variation, and are computed from the beam emittance and the beam lateral profiles at successive positions. Indexes $x$ and $y$ are corresponding to respectively the horizontal and vertical directions. The plain and dashed curves, coming from MAD-X, match exactly HECTOR's output (squares). 


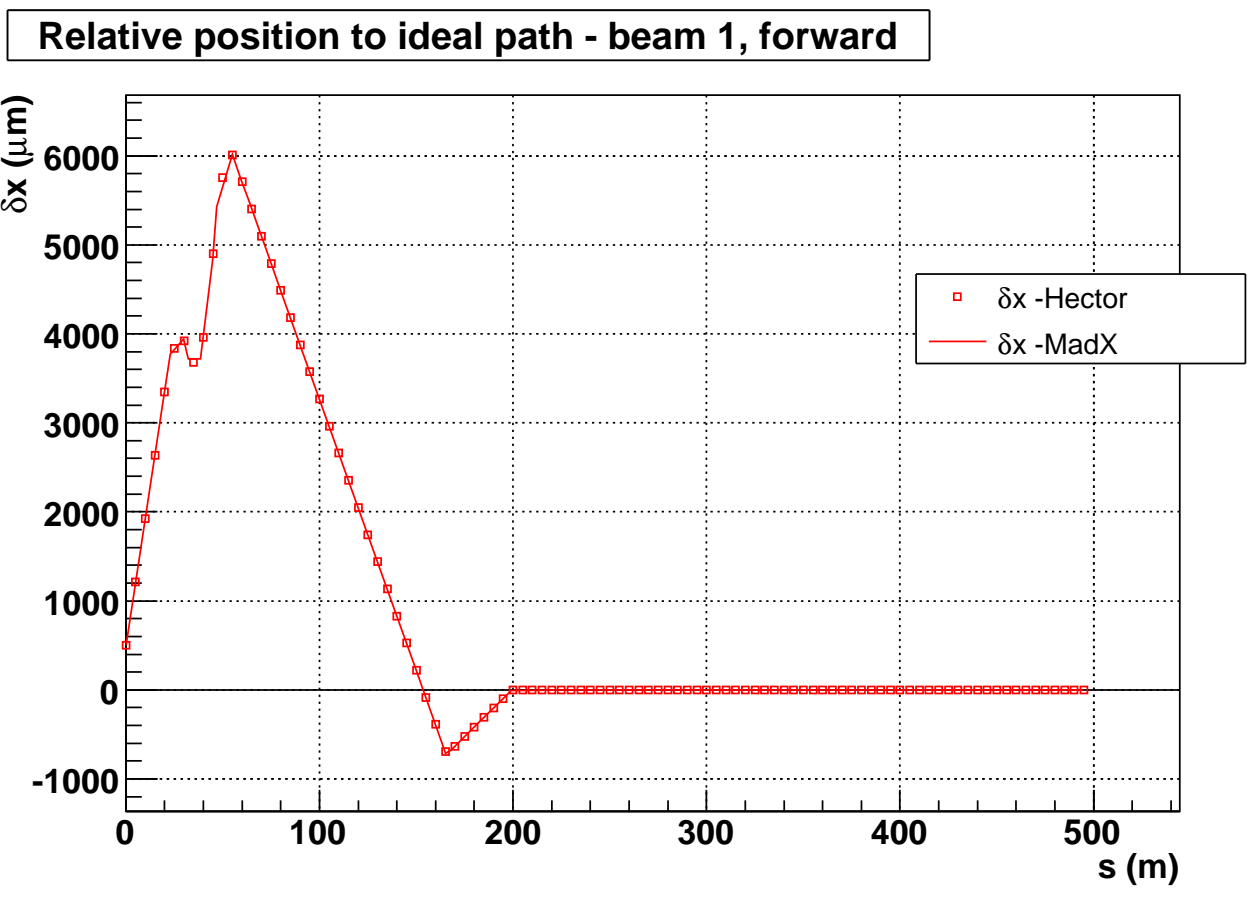

\section{Relative position to ideal path - beam 2, forward}

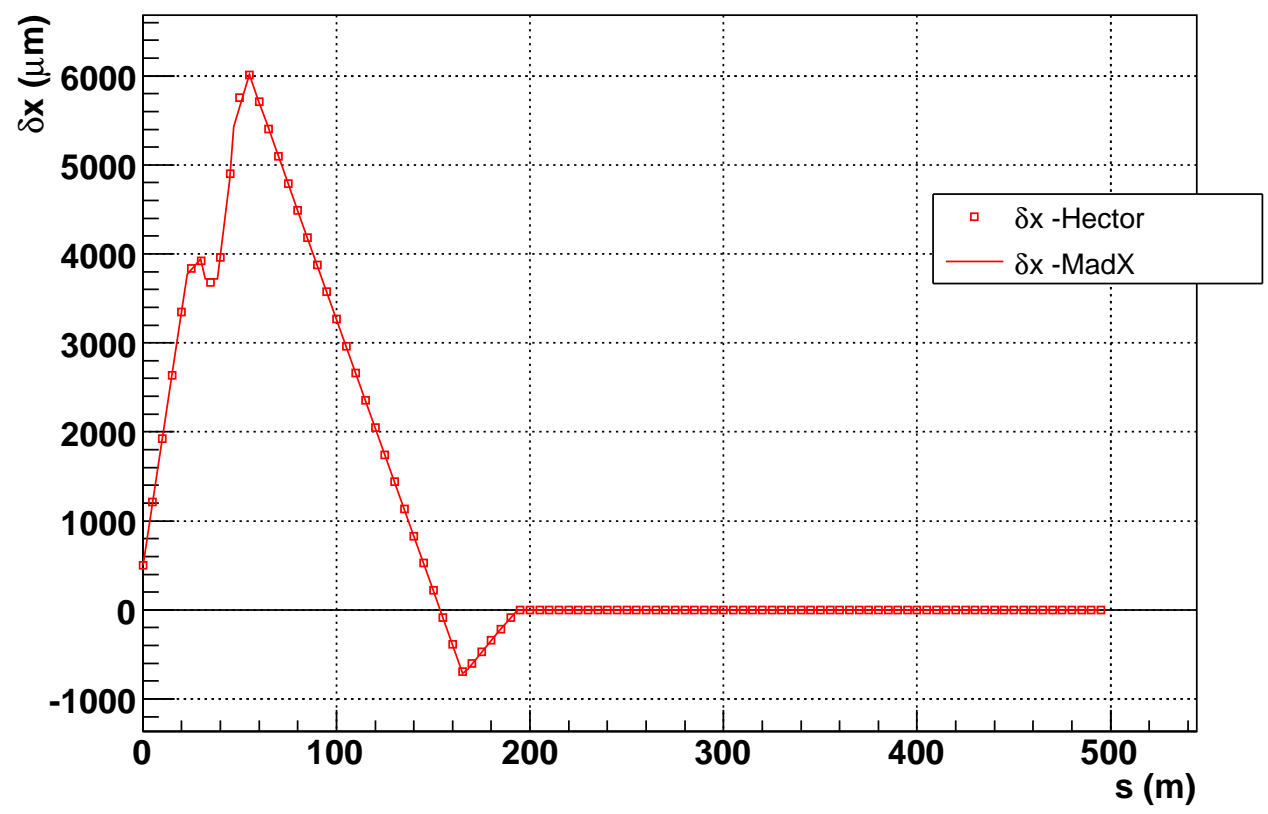

Figure 3: Relative horizontal positions for beams 1 (above) and 2 (below), around the IP5, in the forward direction. The relative position is defined as the difference along the ideal orbit between the beam lateral positions with and without the crossing angle. This relative position graph (squares) matches MAD-X predictions (plain lines). One clearly sees the crossing angle at the IP5 $(s=0 \mathrm{~m})$ and the effects of the kickers that move the beams back to the ideal orbit. 


\section{Beamline simulation}

By using the Hector classes and library physics studies can be performed by means of the particle propagation inside the beamlines, away from the interaction point.

\section{$5.1 \quad$ Trajectories}

Knowing the optics tables for both LHC beams, their trajectories can be compared simultaneously, in both top and side views, for the two LHC beams aside (incoming beam 2 and outgoing beam 1, at the IP5 and IP1: Fig. 4). The top view shows the beams on the horizontal plane, clearly depicting the crossing angle at the IP5, and the beam separation after $70 \mathrm{~m}$ away from the interaction point. The bending of the sector dipoles has been switched off in order to makes graphics more clear - this is why both beams are straight and parallel after $250 \mathrm{~m}$. However, the optical elements have been shifted (without tilt) in the horizontal plane by the half of the beam separation distance, from $180 \mathrm{~m}$ away from the IP, in order to match the ideal beam path: a proton with nominal energy and on the ideal orbit should travel through optical elements in their geometrical center. The side view in turn shows the beams in the vertical plane, emphasizing the difference between IP1 and IP5. In addition, the major optical elements have been drawn: rectangular dipoles in red, sector dipoles in light green, and quadrupoles in yellow and blue.

\subsection{Beam profiles}

Lateral beam profiles along the beamline are of interest, for instance for studies of very forward detectors (Fig. 5). The beam size and shape are obtained after propagation of beam particles using initial dispersion in position, angle and energy at the interaction point. Assuming a Gaussian distribution for these variables, the evolution of both beams can be seen in the transverse plane for example at $220 \mathrm{~m}$ and $420 \mathrm{~m}$ away from the IP. As expected, the beam particles are distributed symmetrically on the vertical plane $(\bar{y}=0 \mathrm{~mm})$, and are already separated in the horizontal plane $(\bar{x}= \pm 97 \mathrm{~mm})$. The symmetry between beam 1 and 2 is striking. The $3 \sigma$ contours (in red) guides the eye for the lateral beam extension. In the angular $\left(\theta_{x}, \theta_{y}\right)$ plane, the beams evolve according to the focusings and the defocusings they undergo, even if the mean value of these angles remains effectively equal to $0 \mu \mathrm{rad}$. The evolution of the beams in the phase space has also been drawn (Fig. 6). Once again, the similitude between both beams is clear, even if they differ from each other by slight details. All these profiles where obtained by simulation of $10^{4} 7 \mathrm{TeV}$ beam protons.

\subsection{Apertures}

The physical aperture of the real optical elements has also to be taken into account. If a particle hits a collimator or a wall of the primary vacuum system, it is assumed to be lost. Each time a particle enters or leaves an optical element, a test is performed to determine if its position matches the element acceptance. This is illustrated in Fig. 7. Protons from the LHC beam 1, around the IP5 have lost some energy, which leads to a deflection with respect to the nominal beam position in $(0,0)$ in the transverse plane. The aperture shape of the MB.B9R5.B1 optical element is the rectelliptical hatched area. The proton energy has been chosen to $110 \mathrm{GeV}$, in order to match the element limiting energy acceptance. Due to the initial smearings, some protons (in black) still pass through the optical element without being stopped. On the other hand, some of them hit the limiting aperture shape and are tagged as stopped protons (in red). In such a case, the particles are considered as lost from the beam, and not propagated any further. 

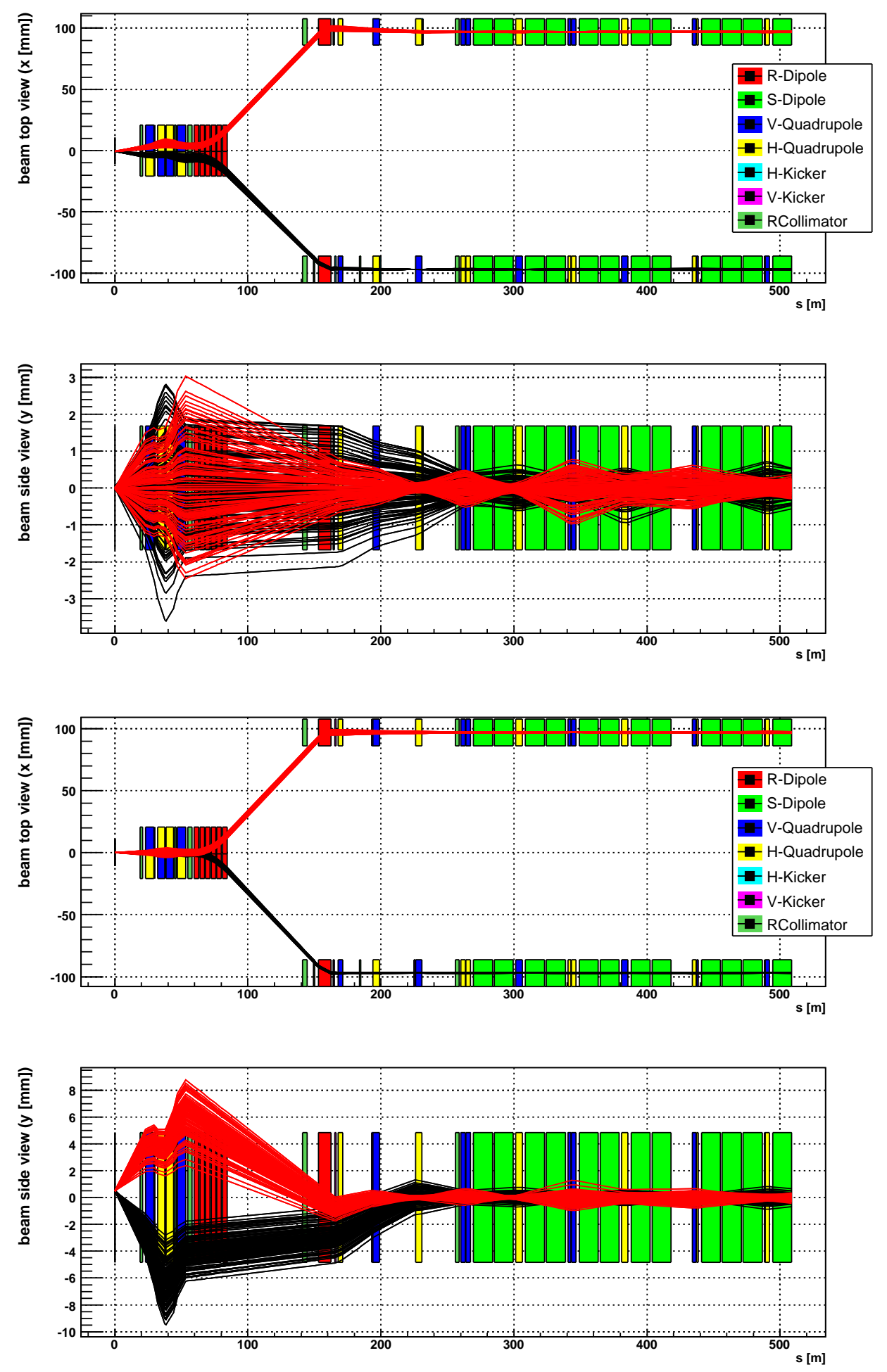

Figure 4: Top and side views of both LHC beams around the IP5 (first two graphs) and at the IP1 (last two graphs). The IP is located at $s=0 \mathrm{~m}$. Beam 2 (red) flows from the right to the left and is seen before its passage at the IP. In turn, beam 1 (black) is seen downstream, passing from the left to the right after the crossing. In this graph, the bending effect of the sector magnets (S-Dipoles) has been switched off, thus rectifying the beam path to a straight line after $250 \mathrm{~m}$. The difference between the IP1 and IP5 is clearly seen, as the crossing planes are perpendicular to each other. Note: In the LHC absolute frame, the position of the IP1 is at $s=0 \mathrm{~m}$ and the IP5 is at $s=13329 \mathrm{~m}$. 

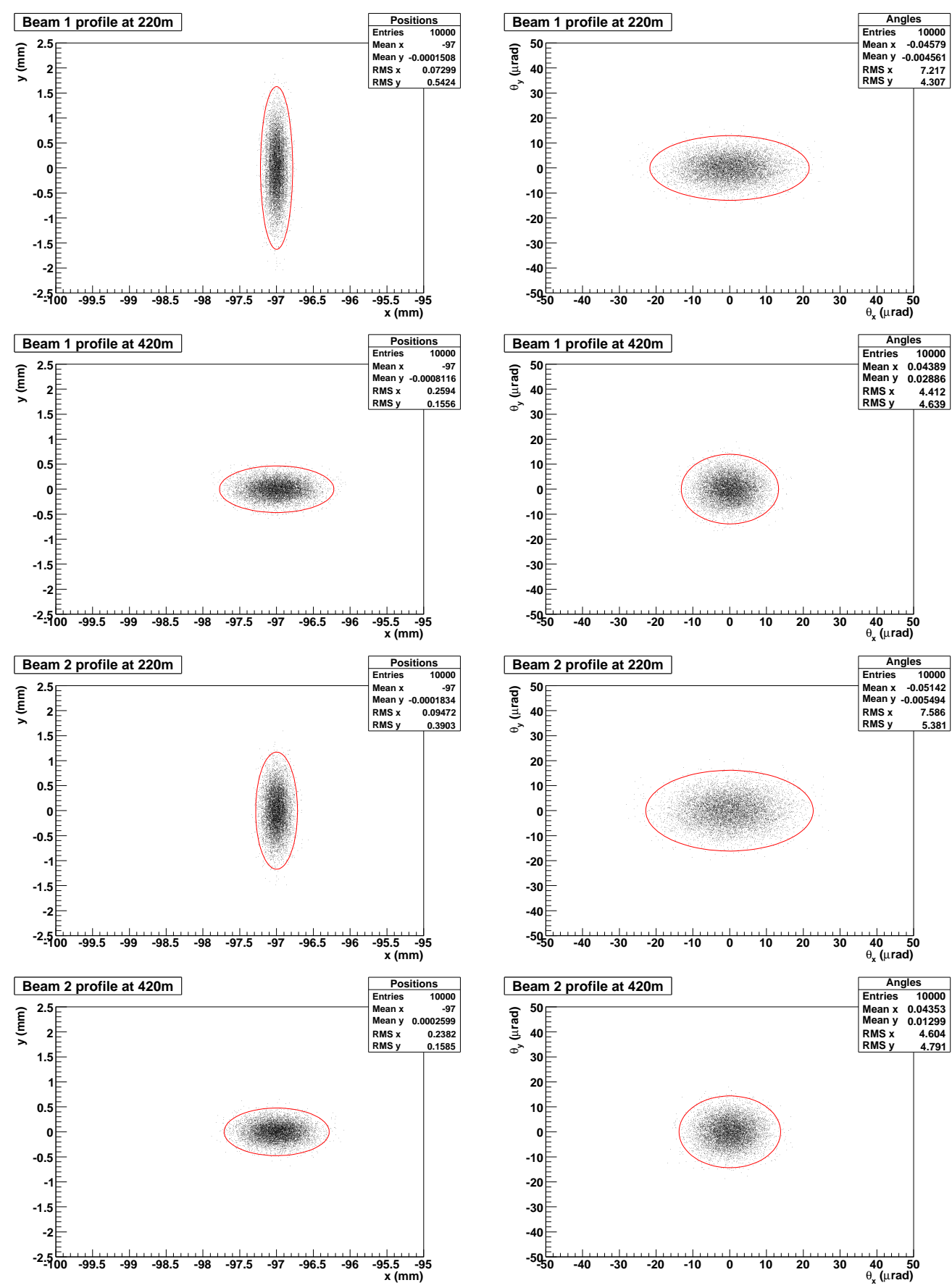

Figure 5: LHC beam profiles in the transverse plane at $220 \mathrm{~m}$ and $420 \mathrm{~m}$ from the IP5, for both beam 1 (above) and 2 (below). The graphs in the left column plot the horizontal and vertical position profiles. The graphs in the right column show the horizontal and vertical angular profiles. These plots are obtained by propagating ten thousand $7 \mathrm{TeV}$ protons through the LHC beamlines, in the forward direction, from the IP5. The mean horizontal position $\bar{x}=-97 \mathrm{~mm}$ matches the half of the separation distance between both beams. The red ellipse delimits the $3 \sigma$ contour for each plots. 

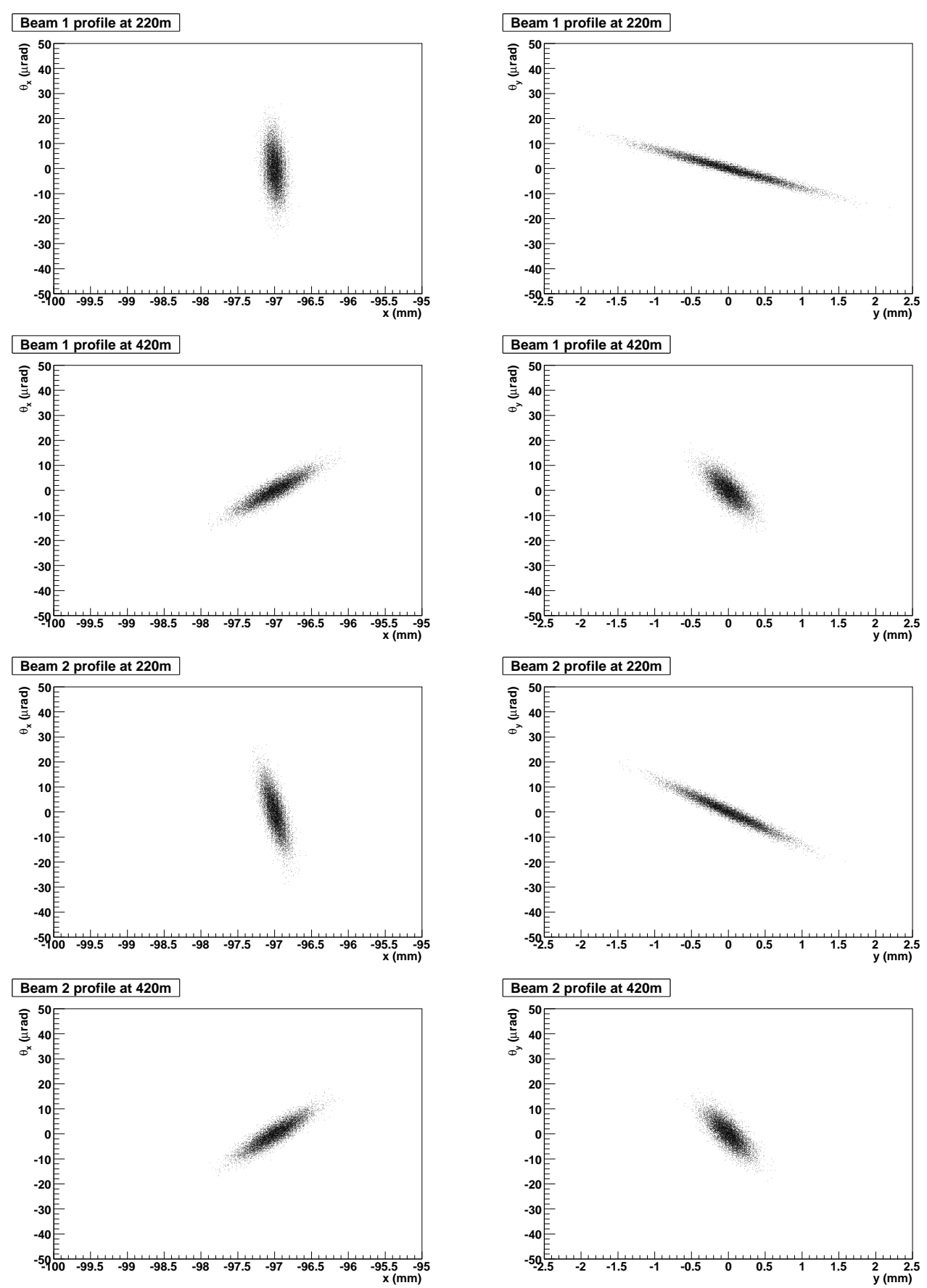

Figure 6: LHC beam profiles in the transverse phase space at $220 \mathrm{~m}$ and $420 \mathrm{~m}$ from the IP5, for both beam 1 (above) and 2 (below). These graphs depict the horizontal $\left(x, \theta_{x}\right)$ and vertical $\left(y, \theta_{y}\right)$ distributions obtained by propagating $10^{4} 7 \mathrm{TeV}$ protons through the LHC beamlines, in the forward direction from the IP5. 
Aperture effect of "MB.B9R5.B1" on $110 \mathrm{GeV}$ energy loss protons

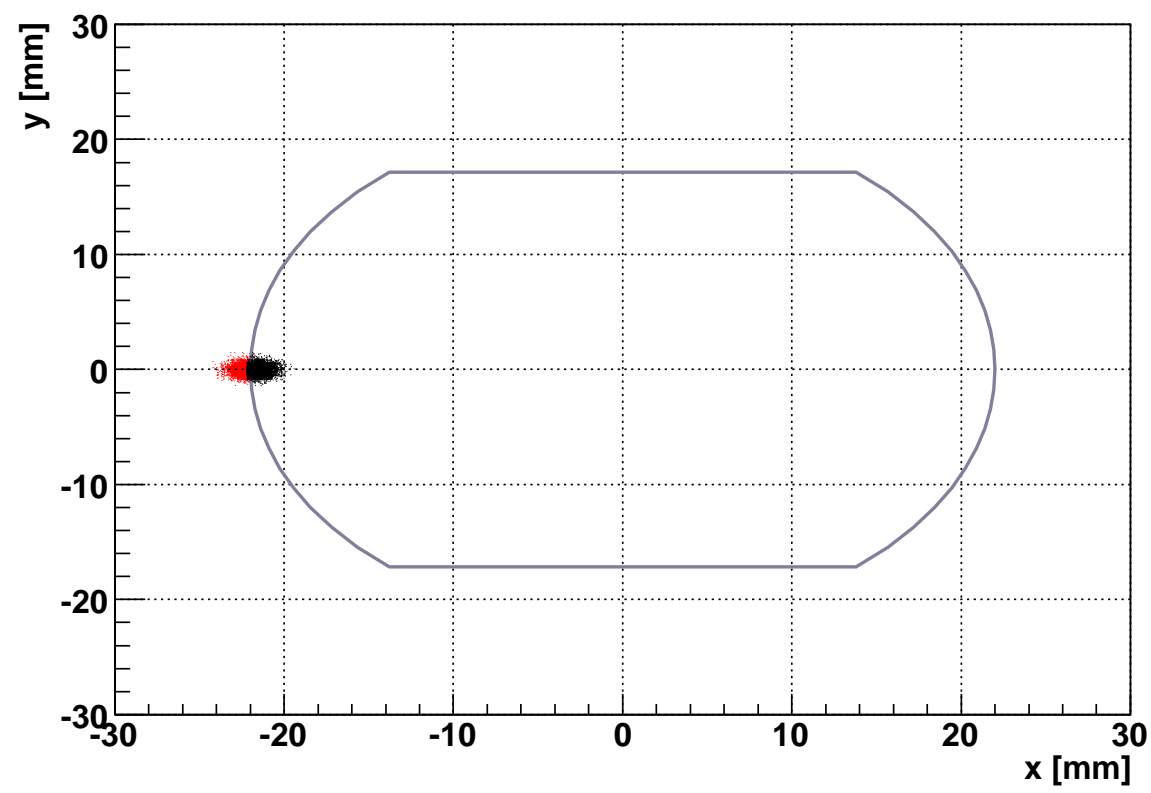

Aperture effect of "MB.B9R5.B1" on $110 \mathrm{GeV}$ energy loss protons

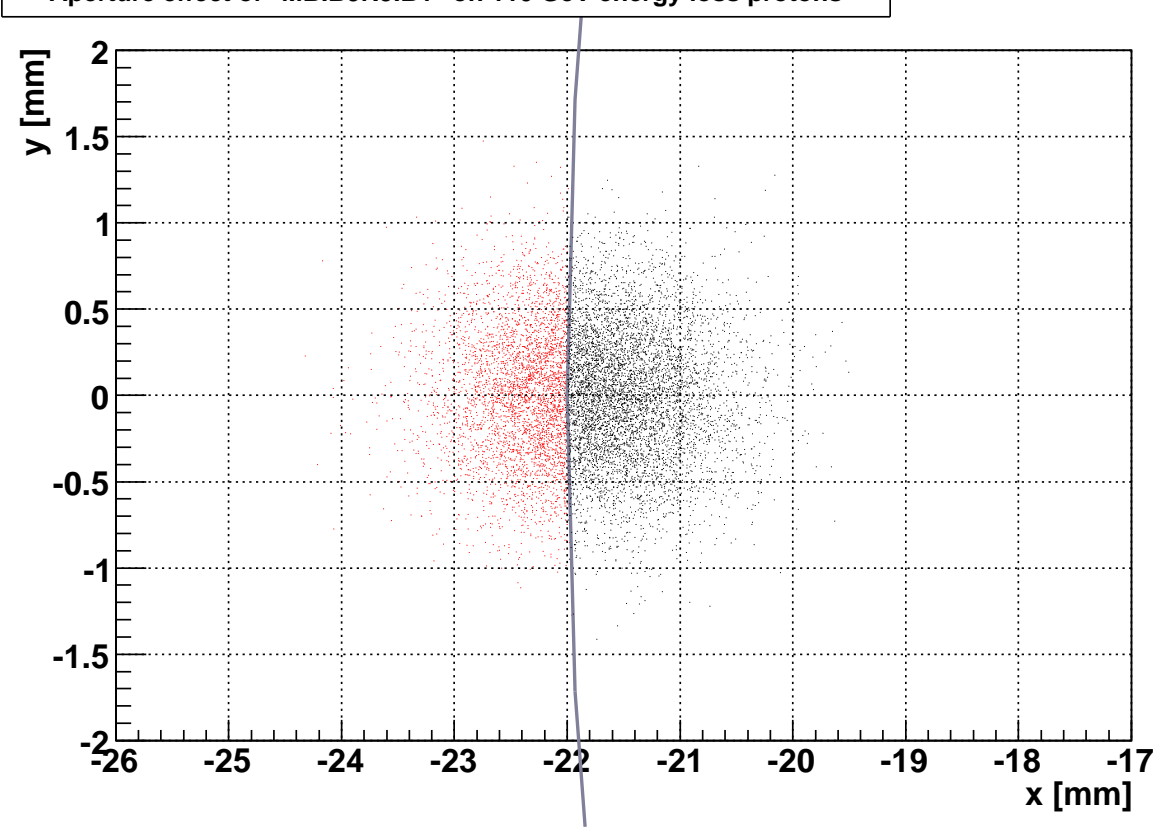

Figure 7: Example of aperture check for the MB.B9R5.B1 dipole $(s=338 \mathrm{~m})$, drawn in the $(x, y)$ transverse plane, at the exit of this optical element. A set of protons, with a mean energy loss around $110 \mathrm{GeV}$, has been propagated from the IP5 to the MB.B9R5.B1 dipole using the LHC beam 1 optics. The beam 1 is centered in $(0,0)$ but is not shown. The closed blue area corresponds to the rectellipse aperture shape of the optical element. Protons that passed through the dipole are the black ones, while protons hitting the walls are tagged in red. The first graph is scaled for the full aperture shape, the second one has been zoomed in around the region of interest. 


\section{$6 \quad$ Very Forward Detectors}

The physics program at the LHC has been extended to processes in which isolated particles at very large (pseudo-)rapidities are produced [6] in diffractive and photon induced interactions [7]. To study those processes, several very forward detectors are being prepared for installation along the beamline. This section will discuss in particular two scenarios, differing by their distance from the IP $(s)$ and their minimal horizontal distance $(x)$ from the nominal beam position. The assumed location of the first detector is $(s=220-224 \mathrm{~m}, x=2000 \mu \mathrm{m})$, and of the second one at $(s=420-428 \mathrm{~m}, x=4000 \mu \mathrm{m})$. No hypothesis is made on their detection efficiency or their resolution, unless quoted. We consider here VFDs providing 2D-measurement ( $x$ and $y$ coordinates), each consisting in fact of two stations separated by 4 and $8 \mathrm{~m}$ as a lever arm for the angle measurement, with no magnetic element in between. In the following several examples of studies performed using HЕCTOR are given.

\subsection{Acceptance}

Using HECTOR's aperture description, it is possible to identify the characteristics of the protons that will hit the VFD. The exchange of a photon or a pomeron, leaving the proton intact, results in a proton energy loss $\left(E_{\text {loss }}\right)$ and a scattering angle, directly linked to the four-momentum transfer squared $(t)$. In case of the photon-induced interactions $-t$ is equal to the virtuality $Q^{2}$ of the exchanged photon. The acceptance windows of the VFDs can be computed by performing scans in $\left(E_{l o s s}, t\right)$ and computing the probabilities of reaching the detectors. The Fig. 8 shows the contour plots of the detectors acceptance, in this $\left(E_{l o s s}, t\right)$ plane. It is obvious from these graphs that the VFD acceptances mostly depend on $E_{l o s s}$, and have a very small sensitivity in $t$, within a large $t$ range. For VFD at $220 \mathrm{~m}$, an area forbidden by kinematics is visible, for low $|t|$ and high $E_{\text {loss }}$. Corresponding profiles at fixed virtuality are shown at $220 \mathrm{~m}$ and 420 m (Fig. 9).

\subsection{Chromaticity grids}

Once the acceptance windows of very forward tracking detectors are defined, it is interesting to see matching between the proton variables at the IP and those measured by VFDs. Depending on their energy and angle at the IP, forward protons will hit the VFDs at various positions. Drawing iso-energy and iso-angle curves for a set of sample protons produces a grid in the measurement related variables, $\left(x_{1}, x_{2}\right)$ or $\left(x_{1}, \theta\right)$. Due to optics of the LHC beamlines, the grid unfolds itself in a much clearer way in the latter plane, and is almost invisible in the former one. The energy dependence of the transfer matrices implies a deformation of the grid - without such a dependence, the grid would be a parallelogram. One should note, that uncertainty of the transverse position of the proton vertex at the IP results effectively in smearing the chromaticity grids. Anyway, these chromaticity grids provide a straightforward tool for unfolding the energy and angle at the IP of the measured particle. The grids in Fig 10 were calculated in the energy range accessible to the VFDs. Finally, the correlation between the vertical angle at the IP and the vertical coordinate measurement is shown in Fig 11.

\subsection{Irradiation levels}

The total diffractive cross-section at the LHC is very large, resulting in a high rate of diffractive protons hitting the VFDs. As a result, it causes extremely high irradiation levels, requiring in turn usage of very radiation hard detectors. As an illustration, the number of hits and their respective map, due to the $\mathrm{pp} \rightarrow \mathrm{pX}$ processes has been investigated (Fig. 12). In this approach, we neglect other radiation sources, like beam halo or secondary particles coming from interactions in the beam pipe for instance.

The pp $\rightarrow$ pX processes are generated by PүтніA [8] 6.2.10 (single diffraction, process number 93). The number of hits are normalized by year by square centimeter, assuming an integrated luminosity of 
$L=20 \mathrm{fb}^{-1}$. From the $10^{6}$ generated events, $17 \%$ have been detected at $220 \mathrm{~m}$, and about $12 \%$ at $420 \mathrm{~m}$. This simulation shows that the hottest spots in the detectors are localized with areas of only a few square $\mathrm{mm}$, reaching proton annual proton fluencies of more than $4 \times 10^{14}$ protons $/ \mathrm{cm}^{2}$.

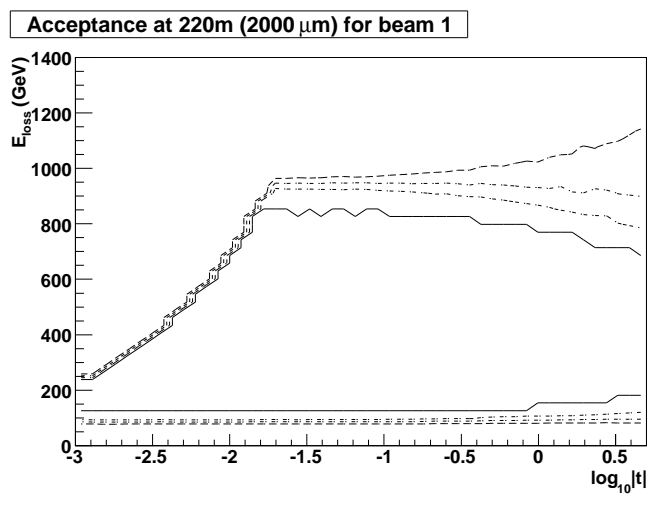

Acceptance at $420 \mathrm{~m}(4000 \mu \mathrm{m})$ for beam 1

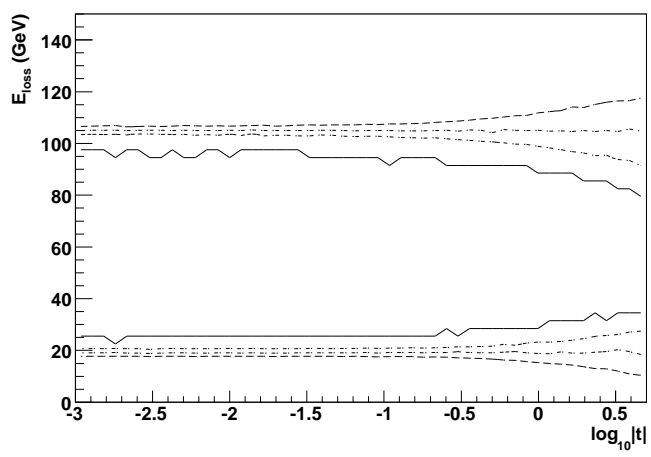

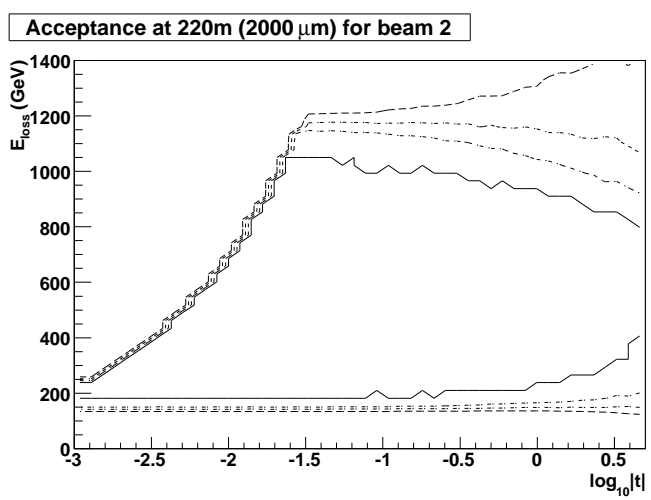

Acceptance at $420 \mathrm{~m}(4000 \mu \mathrm{m})$ for beam 2

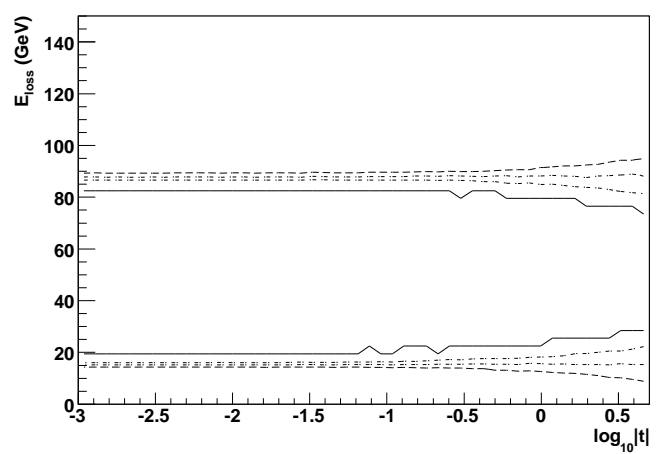

Figure 8: VFD proton acceptance for the LHC beam 1 (left) and 2 (right) around the IP5. These VFDs are located at $(s=220 \mathrm{~m}, x=2000 \mu \mathrm{m}$ horizontal position ; above) and $(s=420 \mathrm{~m}, x=4000 \mu \mathrm{m}$ horizontal position ; below). This map shows contours of 25\%, 50\%, 75\% and (plain curve) 100\% acceptance. The acceptance is roughly rectangular, i.e. independent of $t$. At $220 \mathrm{~m}$, the missing triangle in the acceptance at low $|t|$ and high energy loss is prohibited by kinematics. This area corresponds to non-physical protons. The stair-stepping effect of the lower border of this triangle is only due the binning of the graph. At bottom right corner of each graph, the angular kick coming from the large momentum transfers leads to an increasing smearing of the graph lower edge. 

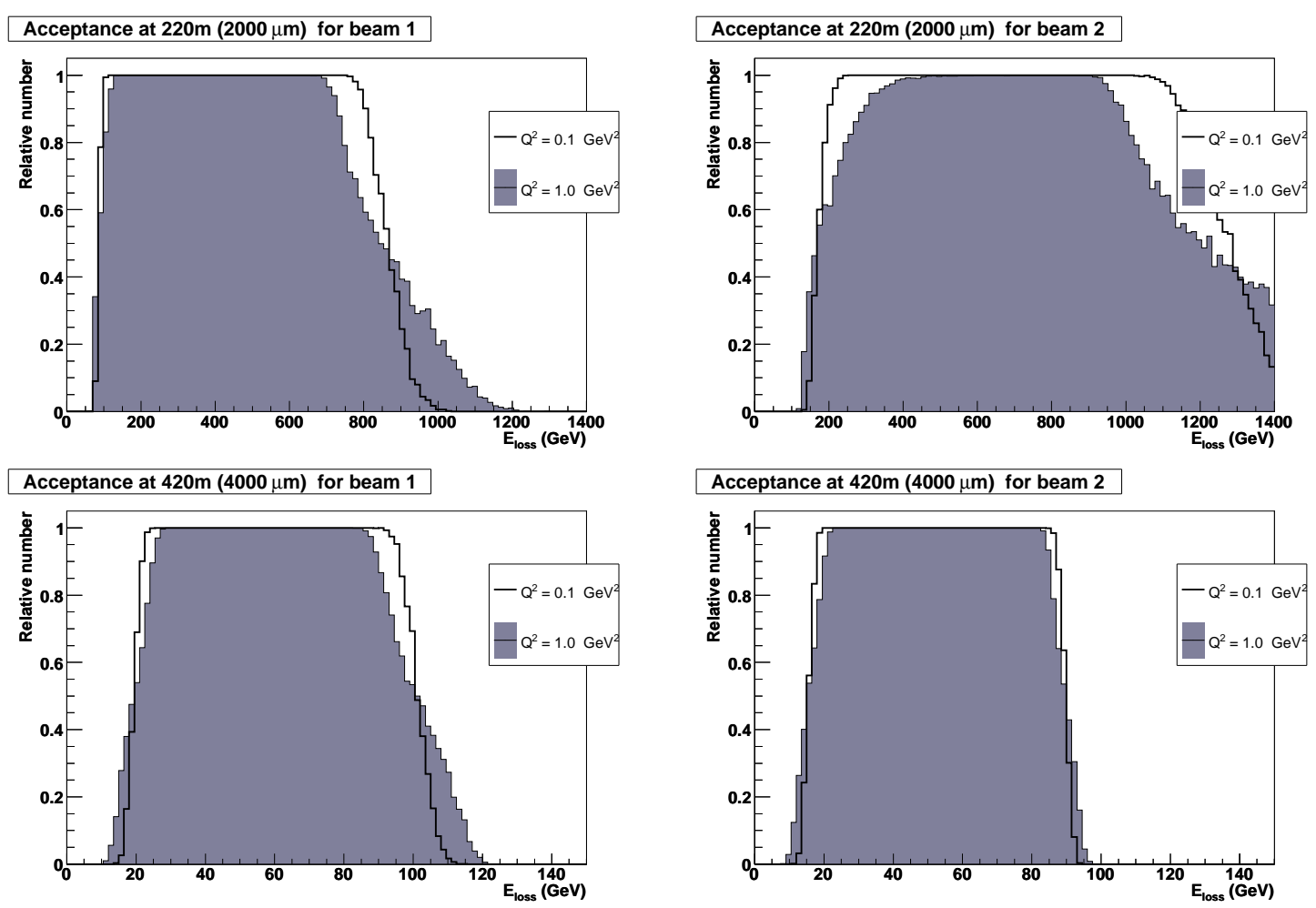

Figure 9: VFD acceptance as a function of energy loss, for two fixed virtualities (Reminder: $Q^{2}=-t$ ). See previous figure for more details. 
Chromaticity grid at $220 \mathrm{~m}$

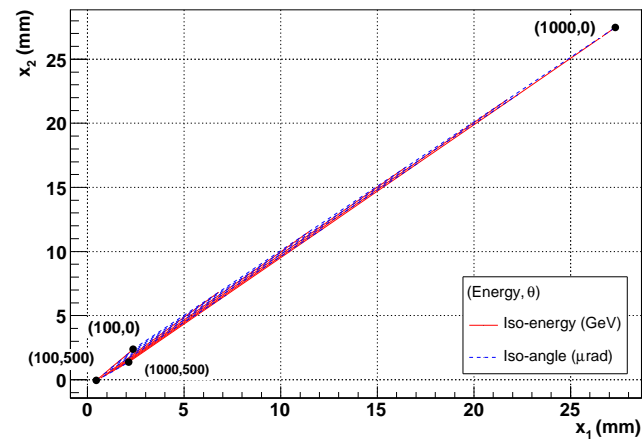

Chromaticity grid at $220 \mathrm{~m}$

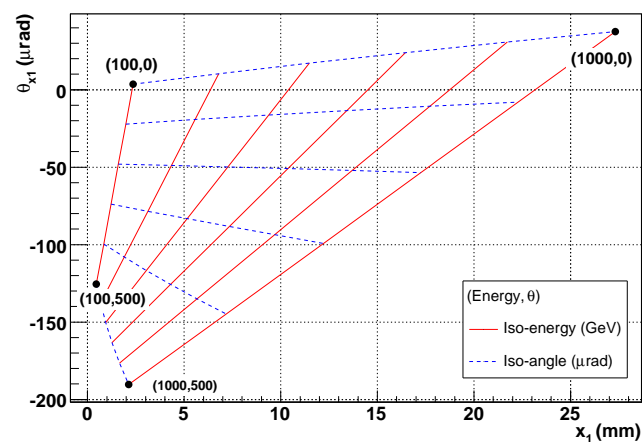

Chromaticity grid at $420 \mathrm{~m}$

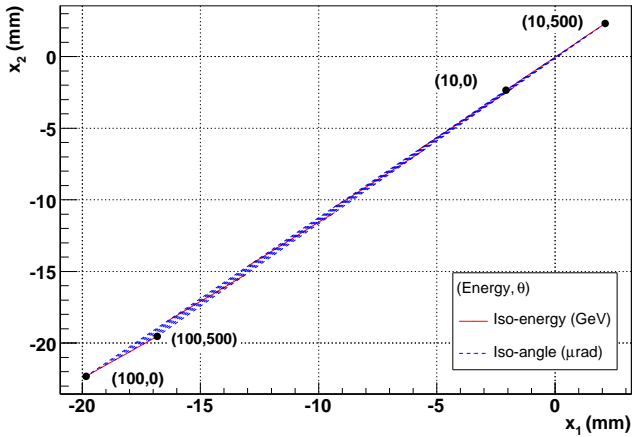

Chromaticity grid at $420 \mathrm{~m}$

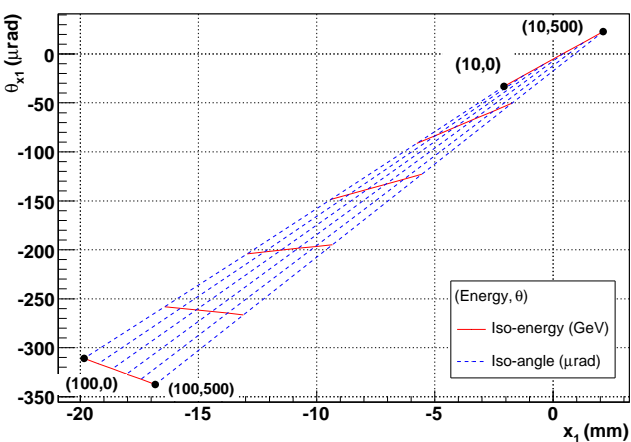

Figure 10: Chromaticity grids: iso-energy and iso-angle lines for the VFDs at $220 \mathrm{~m}$ (left) and $420 \mathrm{~m}$ (right) away from the IP5, for the LHC beam 1 . The graphs show the positions $x_{1}$ and $x_{2}$ of protons, given the energy loss $[0 ; 1000] \mathrm{GeV}$ and $[0 ; 100] \mathrm{GeV}$, respectively, and the angular kick $[0 ; 500] \mu \mathrm{rad}$. The energy dependence of the transfer matrices induces a deformation of the grid, worsening the reconstruction power at higher angles. At $420 \mathrm{~m}$, the grid completely squeezes to a single line. The grids unfold themselves better in the $x-x^{\prime}$ plane.
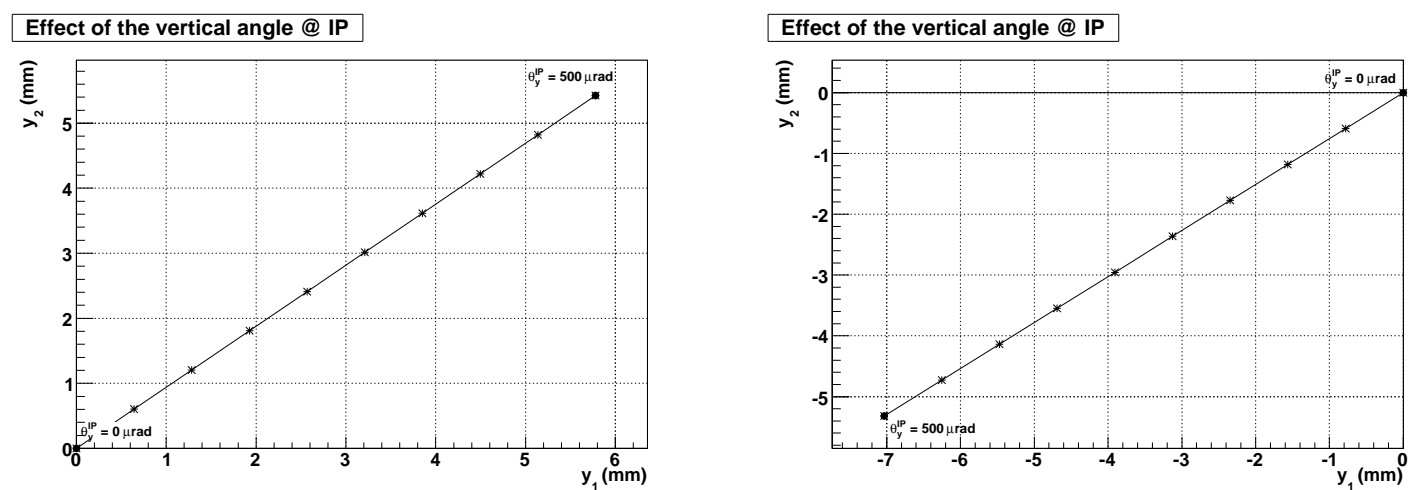

Figure 11: Shift in vertical coordinate $\left(y_{1}\right.$ and $\left.y_{2}\right)$ for VFDs at 220 (left) and $420 \mathrm{~m}$ (right) from the IP5, due to the vertical angle $\theta_{y}^{*}$ at the IP. 

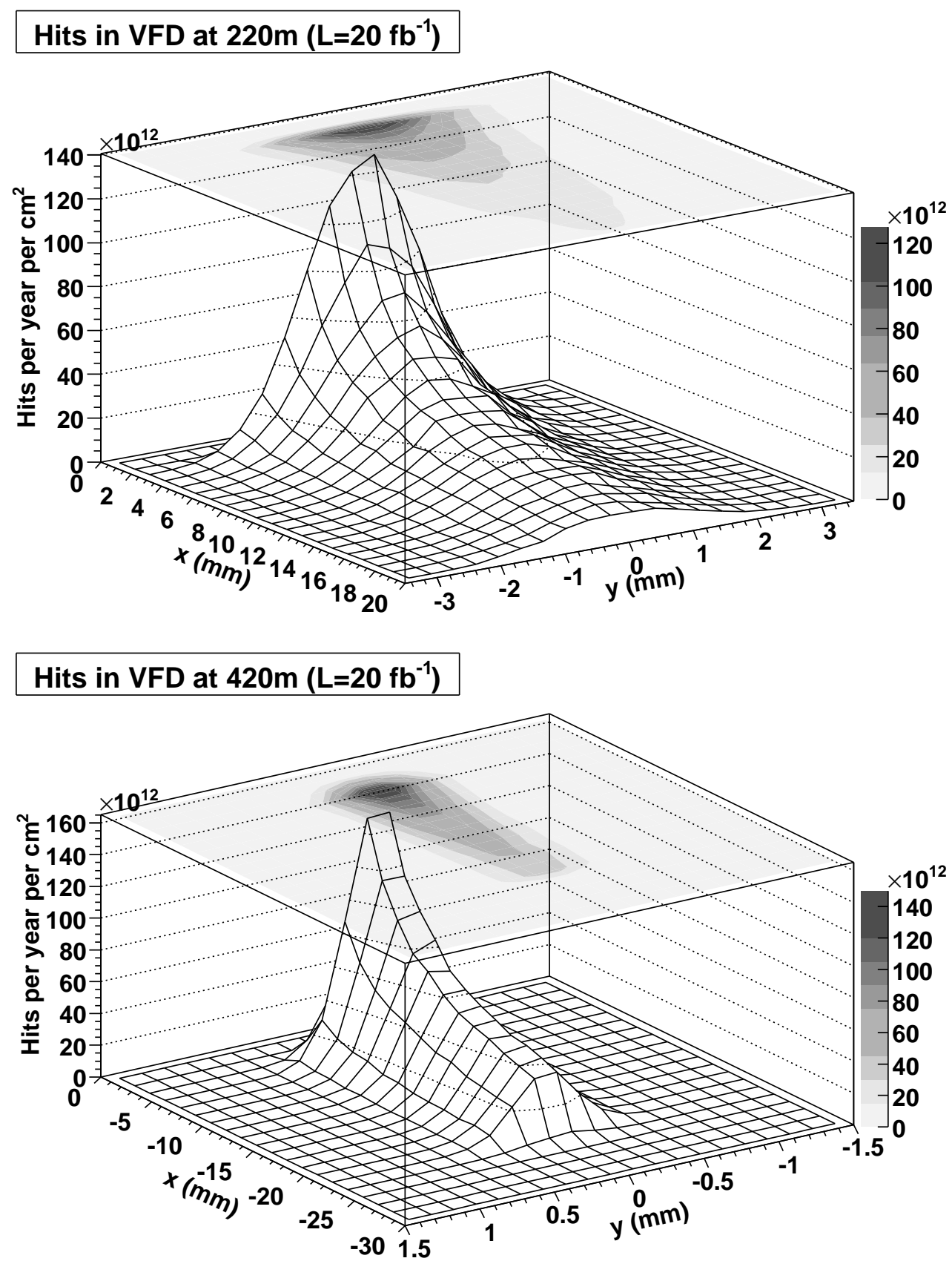

Figure 12: Irradiation levels due to $\mathrm{pp} \rightarrow \mathrm{pX}$ processes, of VFDs located at $s=220 \mathrm{~m}($ above) and $s=420 \mathrm{~m}$ (below) from the IP5. The horizontal position of the detector edge is respectively $x=2 \mathrm{~mm}$ and $x=4 \mathrm{~mm}$ from the nominal position of the center of the corresponding beam. The fluence is given per year and square centimeter. The integrated luminosity of $L=20 \mathrm{fb}^{-1}$ was assumed. For this analysis, HECTOR has propagated protons with 4-momentum generated by PYTHIA 6.2.10 (process number 93). The irradiation levels can locally exceed $1 \times 10^{14}$ protons per square centimeter. 


\subsection{Reconstruction}

One of the main physical motivations for HECTOR is the reconstruction of the event kinematics. For instance, if a beam particle has exchanged a photon at the IP, one could reconstruct photon's energy $(E)$ and virtuality $\left(Q^{2}\right)$. The particle energy at a given position in the beamline is obtained from the measured particle position and angle within the matrix formalism by solving these equations:

$$
\left\{\begin{array}{l}
x_{s}=a_{s} x_{0}+b_{s} x_{0}^{\prime}+d_{s} E \\
x_{s}^{\prime}=\alpha_{s} x_{0}+\beta_{s} x_{0}^{\prime}+\gamma_{s} E
\end{array}\right.
$$

The transfer matrix of the beamline yields the coefficients $a, b$, etc. The introduction of an energy dependence on the strength of optical elements refines the transfer matrix, becoming a function of $E$ : $a_{s}(E)$, $b_{s}(E), \ldots$ This dependence will introduce non linearities. Let us consider two reconstruction methods for the energy. In the first, so-called, trivial method only the dispersion $D=d_{s}$ is considered.

The calculation of the particle energy using a beam transfer matrix corresponding to the nominal 7 $\mathrm{TeV}$ energy (i.e. assuming constant $D$ ) obviously induces some errors. Therefore, in order to compensate for the energy dependence of $D$, a polynomial fit to the average (horizontal) position at a given detector location was done as a function of the particle energy. Then, for a fixed four-momentum transfer (or virtuality $Q^{2}$ ), the energy resolution $\delta E$ is almost independent of energy itself (see Fig. 13), apart from the large $Q^{2}$ values for the detector at $220 \mathrm{~m}$. At the zero-degree angle the energy resolution is about 3 and $1 \mathrm{GeV}$ at $220 \mathrm{~m}$ and $420 \mathrm{~m}$, respectively, but the higher is the scattering angle (or the virtuality), the worse is the energy resolution, as shows Fig. 13. So, if no correction is made due to non-zero scattering angle, the average energy resolution for a typical $Q^{2}$ of $0.3 \mathrm{GeV}^{2}$ is worse, about 5 and $2 \mathrm{GeV}$ at $220 \mathrm{~m}$ and $420 \mathrm{~m}$, respectively. The trivial method is not much sensitive on the position resolution of VFDs only if resolutions are close to the horizontal beam size at a given location (70 and $250 \mu \mathrm{m}$, respectively), they would change significantly the energy resolution. However, in this method neglecting the terms $a_{s} x_{0}$ and $b_{s} x_{0}^{\prime}$ leads to significant sensitivity to the non-nominal values of the average vertex position and beam direction (tilt) at the IP, as shown in Fig. 14. While the average vertex position can be very well measured using the central detectors, the beam tilt is more difficult to control - it should be known to better than 10-20 $\mu \mathrm{rad}$ to avoid causing a significant bias.

Once the energy has been reconstructed (Fig. 13 and 14), the scattering angles at the IP can subsequently be computed, after applying all the usual smearings on other variables. As it can be seen in Fig. 11 , the reconstruction of vertical angle is really good both at 220 and $420 \mathrm{~m}$. Even for very modest vertical position resolutions of $50 \mu \mathrm{m}$, the reconstruction resolutions are already limited only by the vertical beam divergence of about $30 \mu \mathrm{rad}$. This reflects the fact that all dipole fields are horizontal around the IP5, and it is effectively a simple 'geometrical' measurement. Unfortunately, as expected, the situation is much worse in the horizontal plane, where the errors and biases are large and increase with energy loss (see Fig 15). However, in the case of the $220 \mathrm{~m}$ detectors it seems possible to correct for the bias and recover the angular resolution close to the ultimate $30 \mu \mathrm{rad}$. It is much more difficult at $420 \mathrm{~m}$ from the IP this means that $\theta_{x}$ at the IP cannot be well reconstructed at $420 \mathrm{~m}$ with the trivial energy reconstruction method, and possibly could be only used to apply selection cuts.

In the second (advanced) method one reconstructs the energy and scattering angles using position measurements at two detector stations at the same time. This requires to solve the equations for $x_{s}$ at both detectors for E. The $a_{s}, b_{s}$ and $d_{s}$ coefficients of the transfer matrix depend on energy with rather complicated shapes, as they are products of many magnets matrices. One efficient way to get those is then simply to fit each coefficient as a function of energy. Various fitting functions were tried, but a quadratic fit proved to be sufficient to avoid any visible bias or resolution degradation.

Using these fitted coefficients, one can easily get a formula for $x_{0}$ as a function of $x$ at both detectors and of the energy. For each pair of detector $x$ coordinates, the used method is to numerically find the $\operatorname{root}\left(x_{0}=0\right)$ of the formula to get the energy corresponding to a $x_{0}=0$, and thus neglect the interaction point transverse extension. 
This method allows to get reconstructed energy independently of the angle of the particle at the IP. Energy reconstruction resolutions does not degrade anymore if the particle transverse momentum $p_{T}$ rises, but the price to pay is that the detectors resolutions become critical. In particular, it means that the uncertainty of the reconstructed angle at a given detector location should be better than the beam angular divergences there ( 6 and $1.5 \mu \mathrm{rad}$, respectively). The transverse momentum can thus also be computed from the matrix coefficients once the energy has been reconstructed.

Distributions of reconstructed energy and transverse momentum for detectors at $220 \mathrm{~m}$ and $420 \mathrm{~m}$ are shown in Fig. 16. One can see that the reconstructed energy resolution stays very good even with non-negligible initial particle $p_{T}$. Figures 17 and 18 show the $p_{T}$ and energy dependence of resolutions on the transverse momentum and energy, for various detectors resolutions. As expected, the energy resolution is independent of $p_{T}$ but is sometimes sensitive to the energy as expected from the chromaticity grids of Fig. 10. The effect of the detector resolution, which was absent for the simple reconstruction method described previously, is now clearly visible even for an excellent resolution of $5 \mu \mathrm{m}$, especially for detectors at $220 \mathrm{~m}$ from IP.

In summary, the proton angular distribution at the IP affects the most the energy reconstruction. In contrast, the vertex lateral distribution has negligible impact. As a result, the most important beam parameter, which can change run-to-run, is the beam tilt at the IP. In principle, it can be indirectly controlled by the beam position monitors at 220 and $420 \mathrm{~m}$, but more direct tilt measurements are favored, as by using BPMs next to the IP, or by monitoring the direction of neutral particle (photons, neutrons, or neutral pions) production in the zero-degree detectors (ZDDs).

The energy resolution squared of the scattered proton $\sigma_{E}$ can be then approximately decomposed into four terms:

$$
\sigma_{E}^{2}=\sigma_{0}^{2}+\sigma_{v t x}^{2}+\sigma_{\text {ang }}^{2}+\sigma_{\text {det }}^{2},
$$

where the nominal beam energy dispersion $\sigma_{0} \approx 0.8 \mathrm{GeV}$, the contribution due to the vertex spread $\sigma_{v t x} \approx 0.7 \mathrm{GeV}$ at $420 \mathrm{~m}$ and $1-2 \mathrm{GeV}$ at $220 \mathrm{~m}$, the contribution due to the detector resolution, neglecting the angular effects, $\sigma_{d e t}^{2}$ is small for resolutions better than $50 \mu \mathrm{m}$, and the contribution due to the proton non-zero angle at the IP, $\sigma_{\text {ang }}$, which is very sensitive the angular reconstruction at the VFDs; even for a zero-degree scattering if one neglects effects due to the beam angular divergence at the $\mathrm{IP}, \sigma_{\text {ang }} \approx 1 \mathrm{GeV}$ at $420 \mathrm{~m}$ and $\approx 3 \mathrm{GeV}$ at $220 \mathrm{~m}$. 

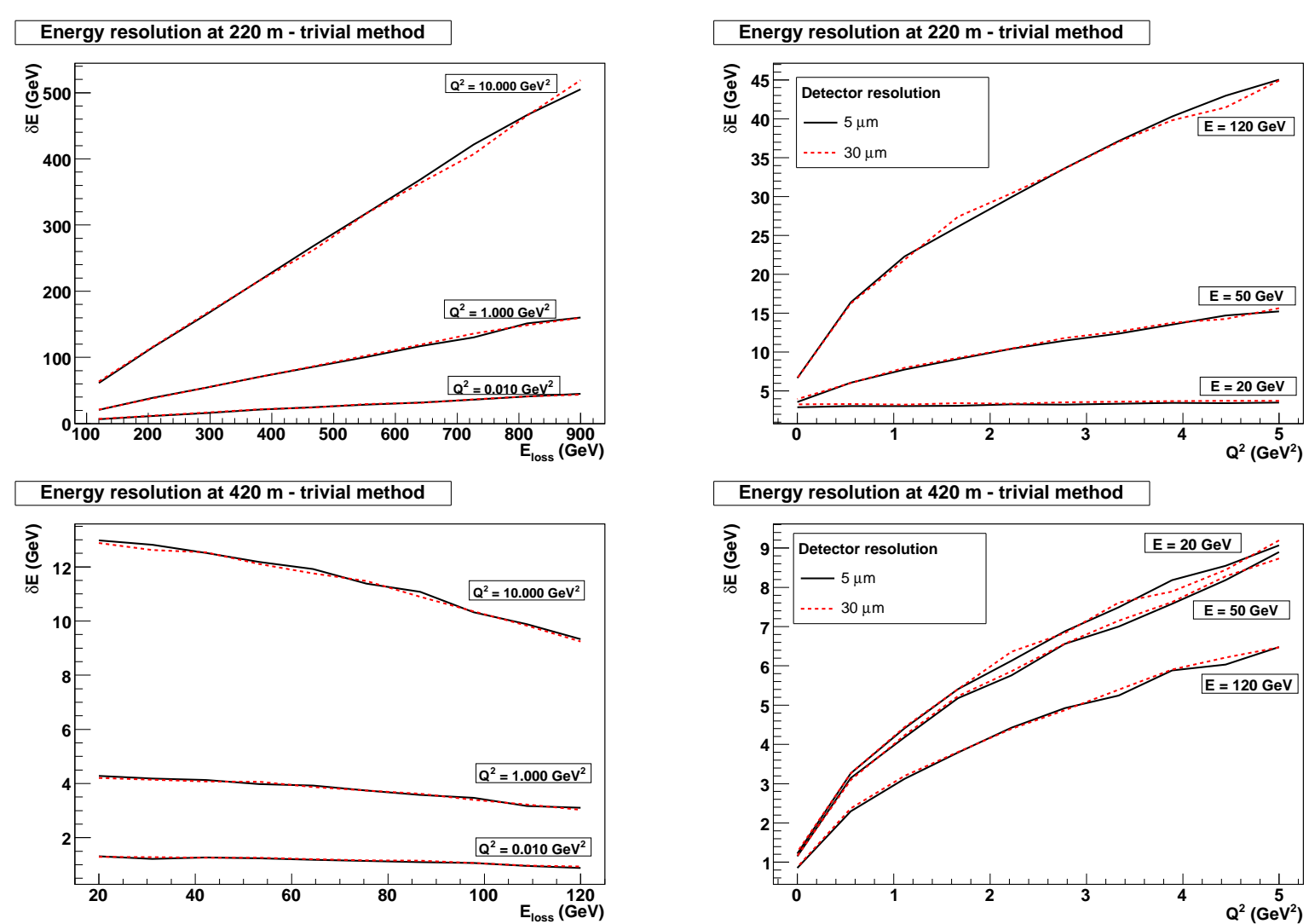

Figure 13: Energy resolution for the trivial reconstruction, for protons measured at $220 \mathrm{~m}$ and $420 \mathrm{~m}$ from the IP5. The protons have exchanged a particle taking away some energy, at a given virtuality. The resolution is shown as a function of the energy loss and the virtuality. The effects of some error on position measurement, due for instance to the spatial resolution of the detector stations ( $5 \mu \mathrm{m}$ and $30 \mu \mathrm{m})$, are taken into account. The beam energy dispersion is not included. 


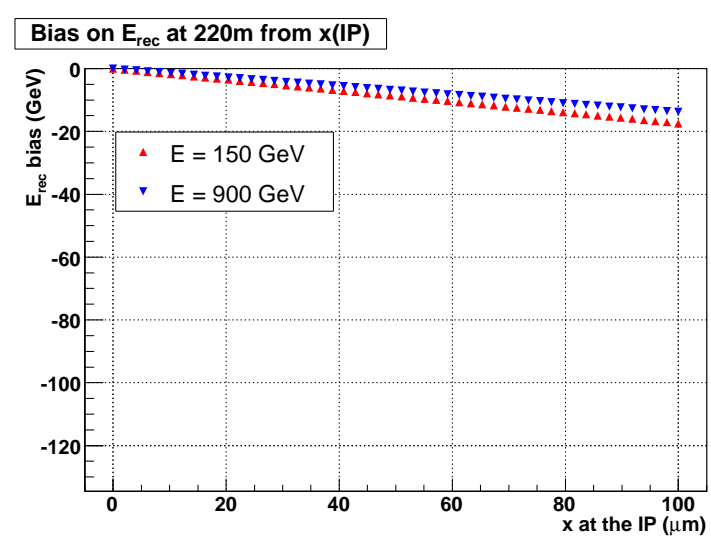

Bias on $E_{\text {rec }}$ at $420 m$ from $x(I P)$

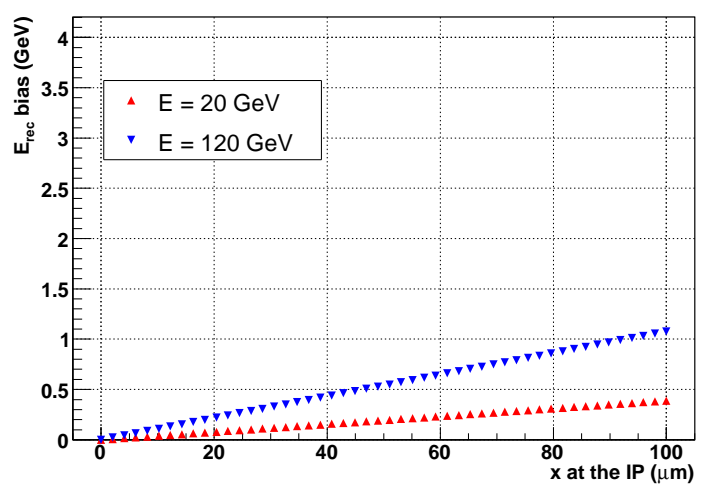

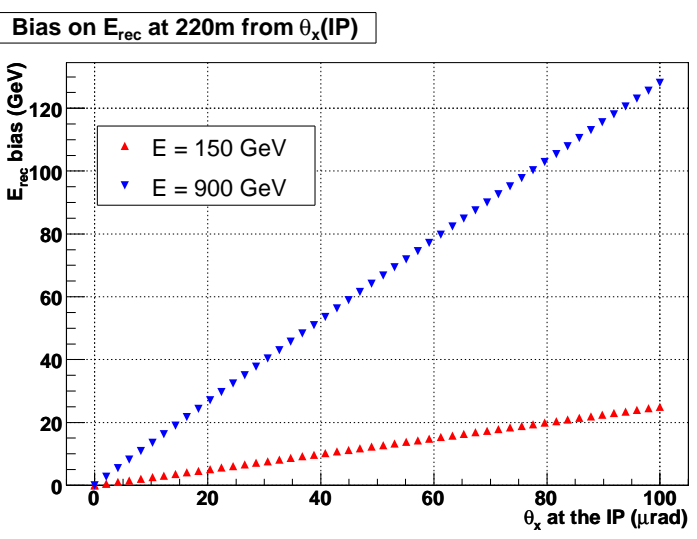

Bias on $E_{\text {rec }}$ at $420 \mathrm{~m}$ from $\theta_{\mathbf{x}}(\mathrm{IP})$

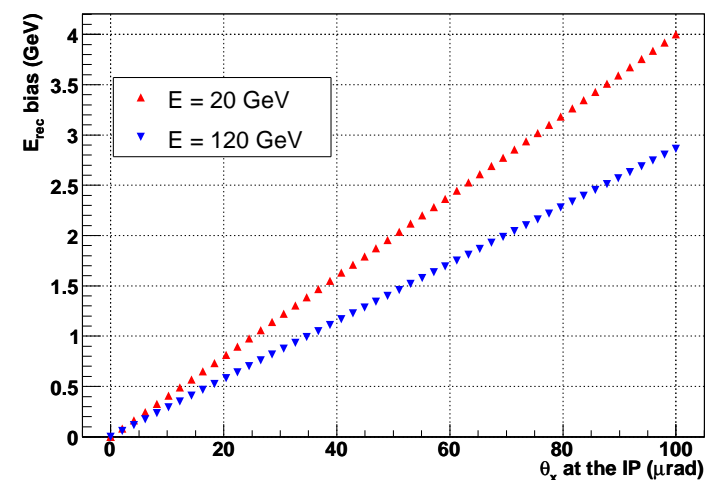

Figure 14: Bias studies for the trivial energy reconstruction for protons measured at $220 \mathrm{~m}$ (above) and $420 \mathrm{~m}$ (below). As the energy is computed using only the dispersion term of the global transport matrix, any non nominal values of the event vertex $x^{*}$ and the beam tilt $\theta_{x}^{*}$ at the IP, results in a biased energy reconstruction. 


\section{Horizontal angle reconstruction @ 220m (100 $\mu \mathrm{rad})$}

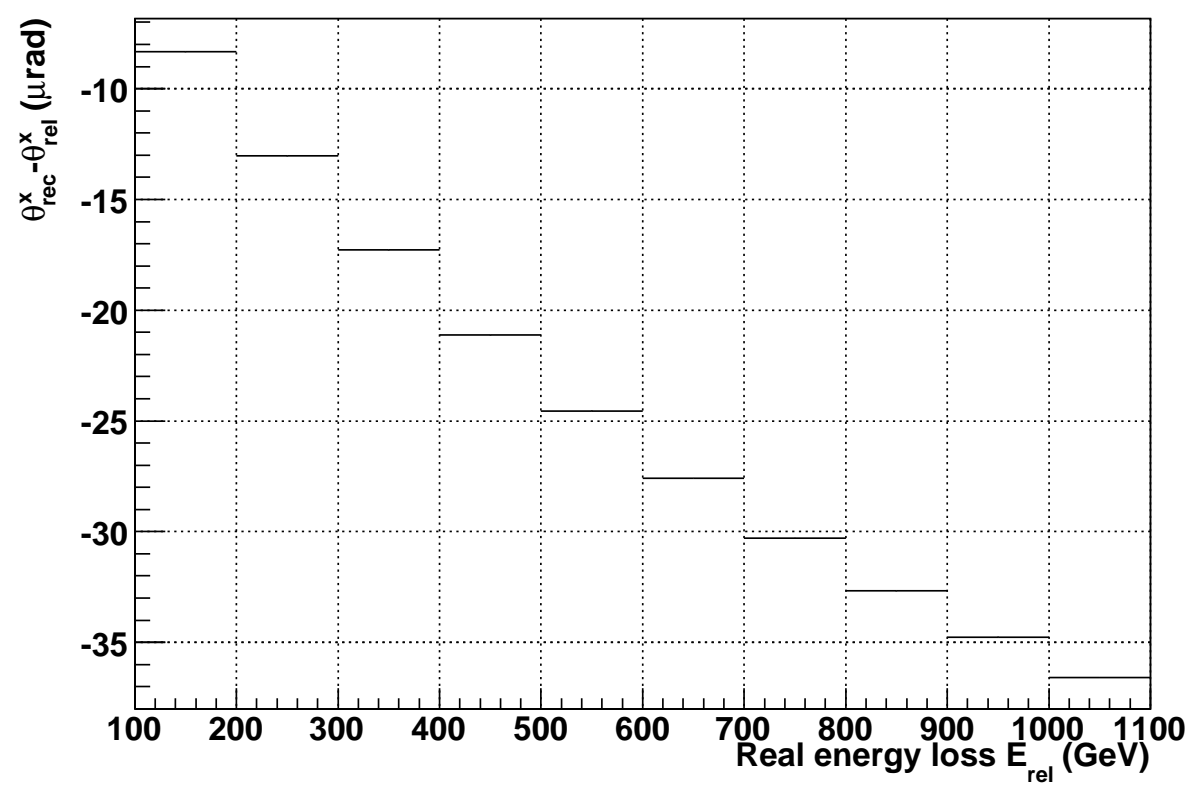

\section{Horizontal angle reconstruction @ 420m (100 $\mu \mathrm{rad})$}

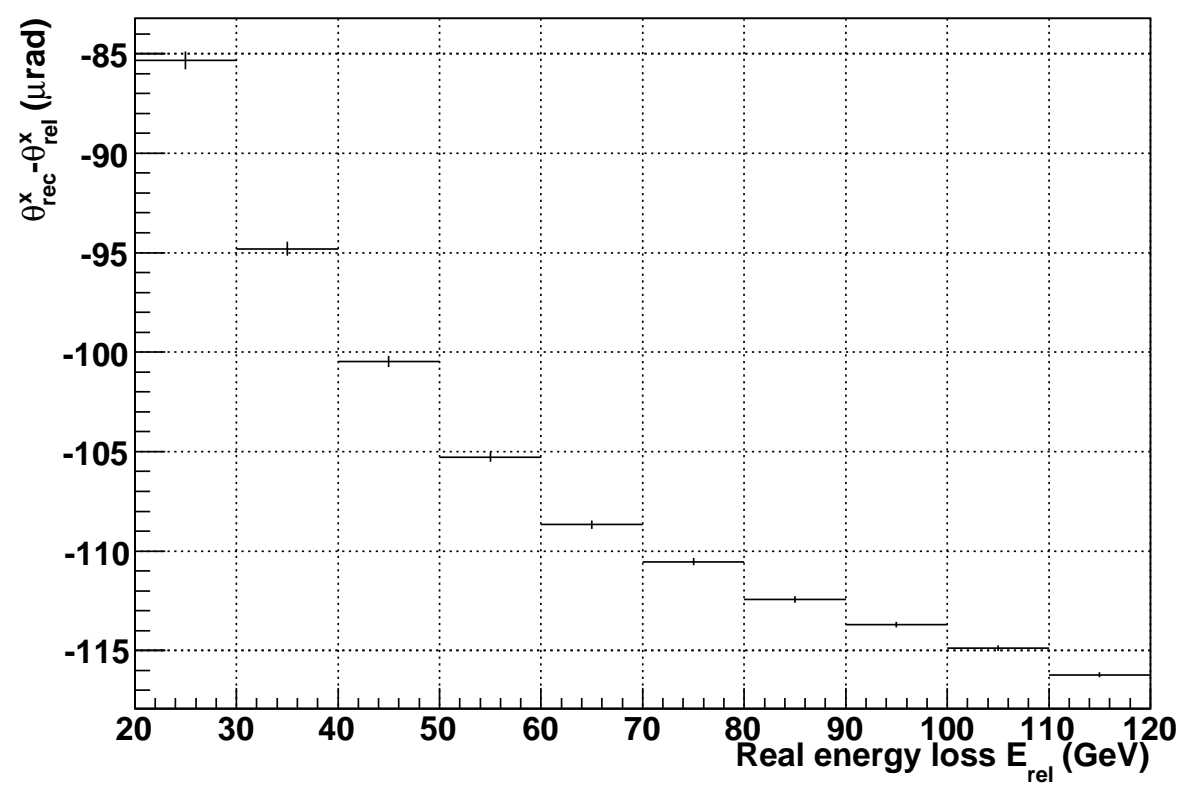

Figure 15: Resolution of the reconstruction of the horizontal $\left(\theta_{x}\right)$ scattering angles, for a $100 \mu \mathrm{rad}$ scattering angle as a function of proton energy loss, for VFDs at 220 (upper plot) and $420 \mathrm{~m}$ (lower plot) from IP5. The trivial energy reconstruction method is used, and the beam divergence at the IP is not taken into account. 

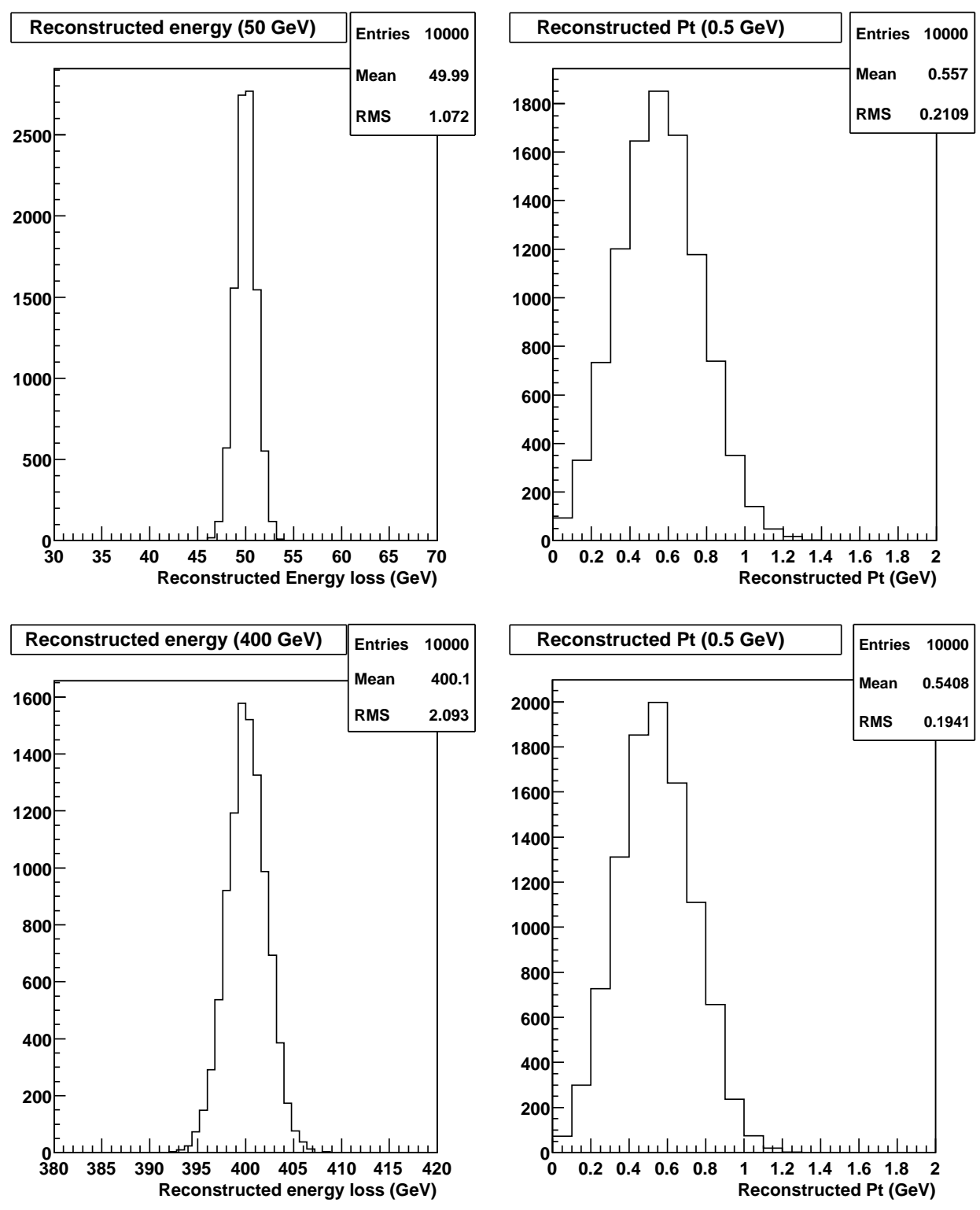

Figure 16: The so-called 'advanced method 'allows to reconstruct at the same time the energy and transverse momentum (or scattering angle). Here examples are given for detectors at $420 \mathrm{~m}$ (upper plot) and $220 \mathrm{~m}$ (lower plot) from the IP, corresponding to energy loss of respectively 50 and $400 \mathrm{GeV}$, and transverse momentum of $0.5 \mathrm{GeV}$ (uniformly distributed in azimuthal angle). Ideal detector is assumed. 

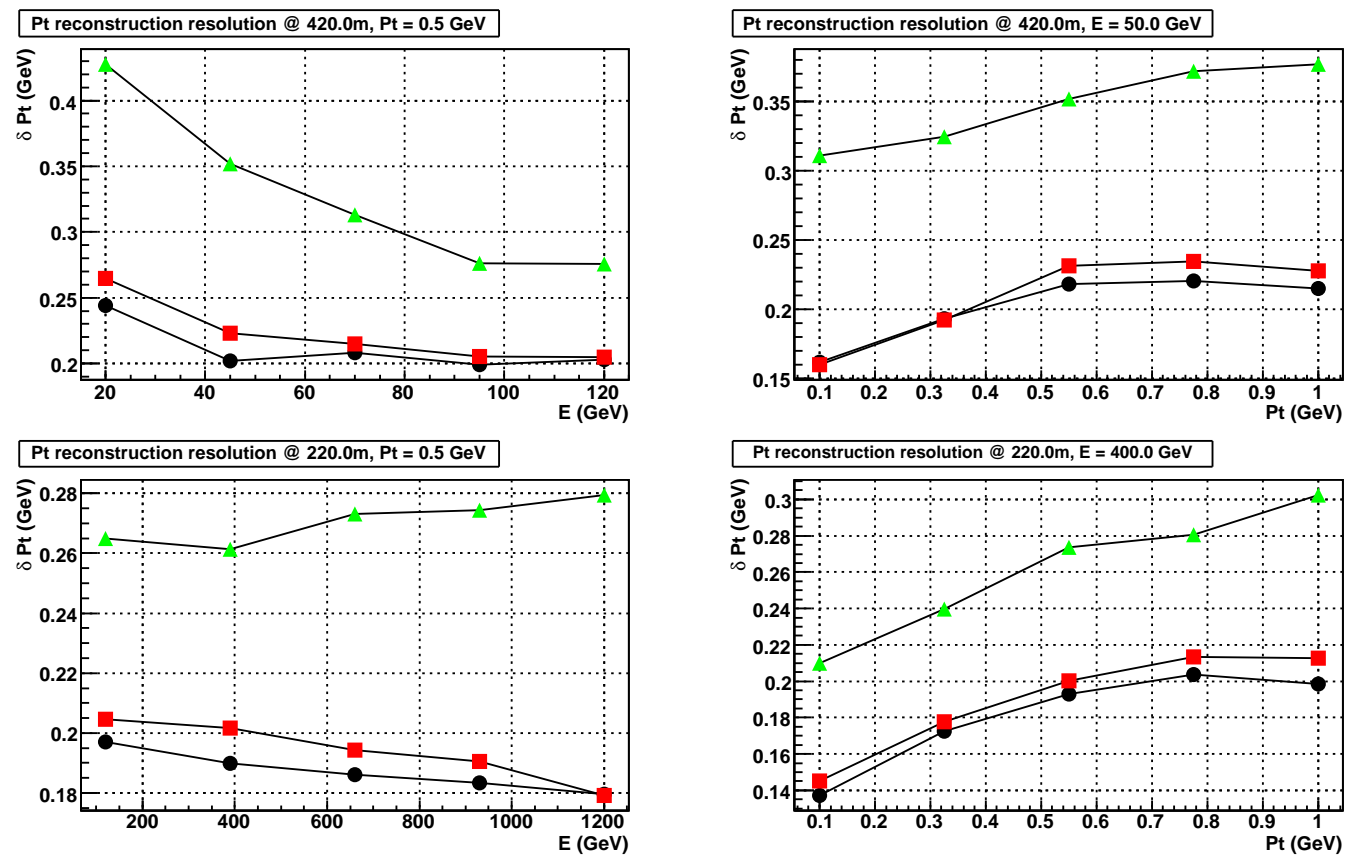

Figure 17: Resolution of the reconstruction of the particle transverse momentum $p_{T}$, as a function of the energy loss (left) and of the transverse momentum (right), for VFDs at 420 (upper plot) and $220 \mathrm{~m}$ (lower plot) from IP5. The advanced method, using full detector information, is used. Dots correspond to different scenarios of detectors resolutions, namely perfect detectors (circles), $5 \mu \mathrm{m}$ (squares) and $30 \mu \mathrm{m}$ (triangles) spatial resolution.
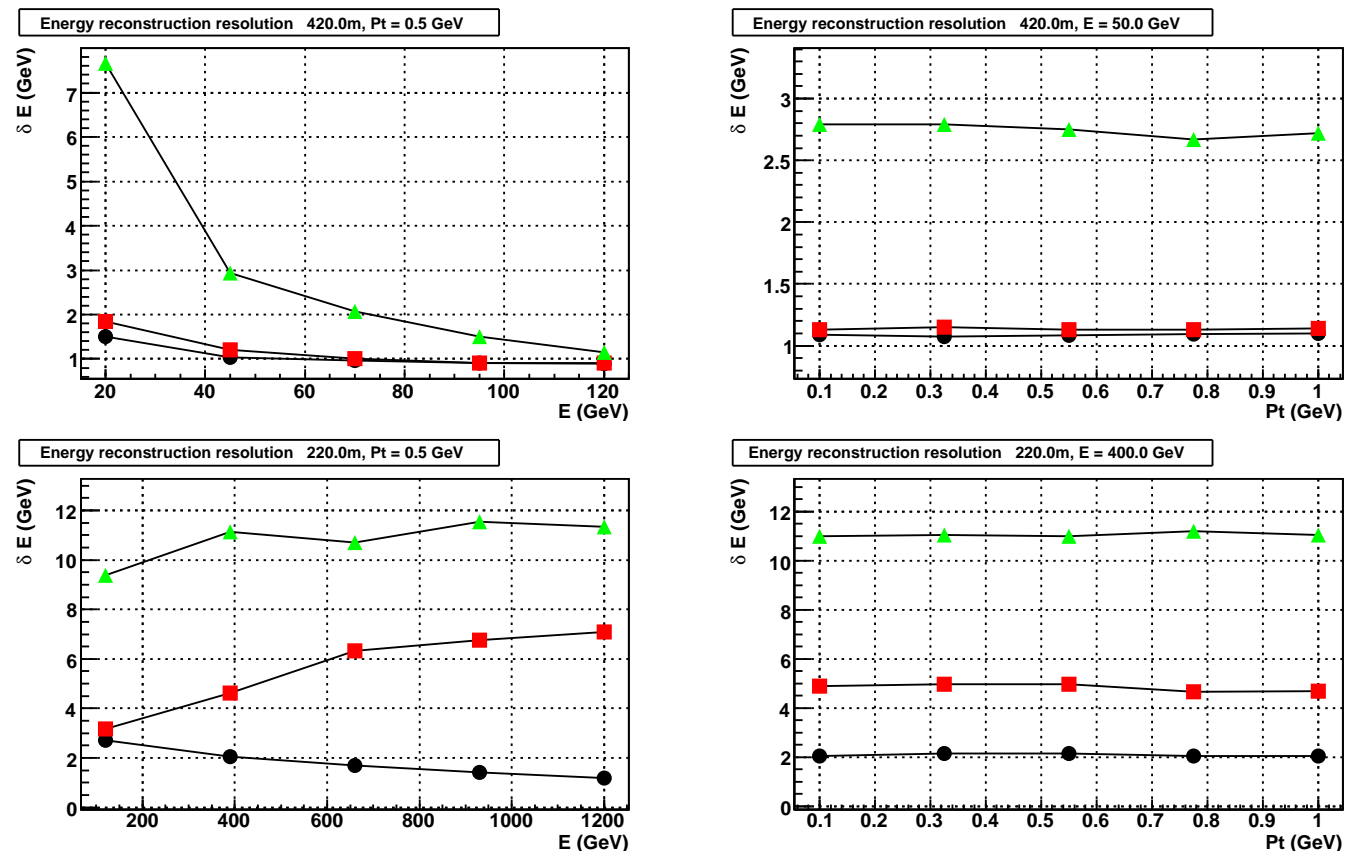

Figure 18: Resolution of the reconstruction of the particle energy loss $E$, as a function of the energy loss (left) and of the transverse momentum (right), for VFDs at 420 (upper plot) and $220 \mathrm{~m}$ (lower plot) from IP5. The advanced method, using full detector information, is used. Dots correspond to different scenarios of detector resolutions, namely perfect detectors (circles), $5 \mu \mathrm{m}$ (squares) and $30 \mu \mathrm{m}$ (triangles) spatial resolution. 


\subsection{Calibration}

Usually, the calibration of the reconstructed variables at forward detectors can be well maintained using the physics processes and the central detectors. At HERA, for example, the elastic $\rho$ meson photoproduction was used where the momentum of the scattered proton could be deduced from two decay charged pions using the central tracking. At the LHC the two-photon exclusive production of dimuon pairs seems a good calibration process, at least for the detectors at $420 \mathrm{~m}$. The visible cross section is large $\left(\sigma_{\mathrm{pp}} \leq 7 \mathrm{pb}\right)$, including the acceptance of central detectors. This should even allow for a run-by-run calibration of the scattered proton energy scale within a full acceptance range. Finally, the expected reconstruction power of central detectors is excellent for such di-muon events, giving the proton energy uncertainty about $10^{-4}$ per event [6]! One should note, however, that using this process it is not possible to check the angular reconstruction, and that it has much more limited statistics within acceptance of the detectors at 220 $\mathrm{m}$. The high-energy bremsstrahlung $p p \rightarrow p p \gamma$ is a possible candidate for the energy scale calibration of the VFDs at $220 \mathrm{~m}$. It has relatively large cross-section, about $10 \mathrm{nb}$ for $E_{\gamma}>100 \mathrm{GeV}$, and photons are emitted into a very forward cone, so could be detected in the zero-degree detectors (ZDDs) at about $100 \mathrm{~m}$ from the IP, proposed for measurements of neutral particles produced at very small angles. A simultaneous measurement of the scattered proton in the VFD and of the bremsstrahlung photon in the ZDD would allow then for cross-calibration of these two devices. In addition, these photons are emitted at very small angles (typically, at less than $150 \mu \mathrm{rad}$ ), so could be used to control the beam direction (tilt) at the IP.

Finally, one should add that the angular measurements could be controlled by studying the reconstruction of the beam angular divergence at the IP, and comparing the obtained results with the direct measurements at the ZDDs, or with estimates obtained using the LHC instrumentation, or derived from the LHC luminosity.

\subsection{Misalignments}

The misalignment of LHC optical elements could have a significant impact on the measurements with very forward detectors. As the deflection of the particle paths depends on their positions in quadrupoles, a misplacement of these optical elements implies a change in the nominal beam position. In turn, as the accurate position measurement with the forward tracking detectors (as well as the information inferred from the segmentation of forward calorimeters) is referred to the ideal beam location, changing this reference results in a biased reconstruction of the measured particles.

Fig. 19 shows the impact of possible shifts $(0.5 \mathrm{~mm})$ and tilts $(0.1 \mathrm{mrad})$ of the beamline quadrupoles on the energy reconstruction with VFDs at $420 \mathrm{~m}$. The reconstruction assumes ideal beamline in which only one quadrupole at a time is separately moved. Effects even higher than $10 \%$ could be expected. The closer to the IP (i.e. the further from the VFDs), the more insensitive the correction. One can partially correct for these effects using information from the beam position monitors, but better results are obtained using a physics calibration process like the two-photon muon pair exclusive production, at least in case of the VFDs at $420 \mathrm{~m}$.

These misalignment effects and the corrections are illustrated (Fig. 20) by the study of two-photon exclusive production of the SM Higgs boson $(\mathrm{pp} \rightarrow \mathrm{pp}(\gamma \gamma) \mathrm{H})$ with $\mathrm{M}_{\mathrm{H}}=115 \mathrm{GeV}$. The measurement of the energy of two scattered protons straightforwardly yields the boson mass, by means of the so-called missing mass method. As a consequence, an uncorrected measurement with misalignment leads to a bad mass calculation. A quadrupole (MQM9R5, $s=347 \mathrm{~m}$ ) close to the detector has been shifted by $100 \mu \mathrm{m}$, and another quadrupole (MQXA1R5, $s=29 \mathrm{~m}$ ) close to the IP by $500 \mu \mathrm{m}$; in the latter case, the misalignment-induced change in the VFD acceptance is visible. The limitations of the beam-positionbased corrections are clearly visible, even assuming no systematic errors, while the muon-calibration stays unbiased (though only a relatively small sample of 700 dimuon events was used to get the correction factors). 

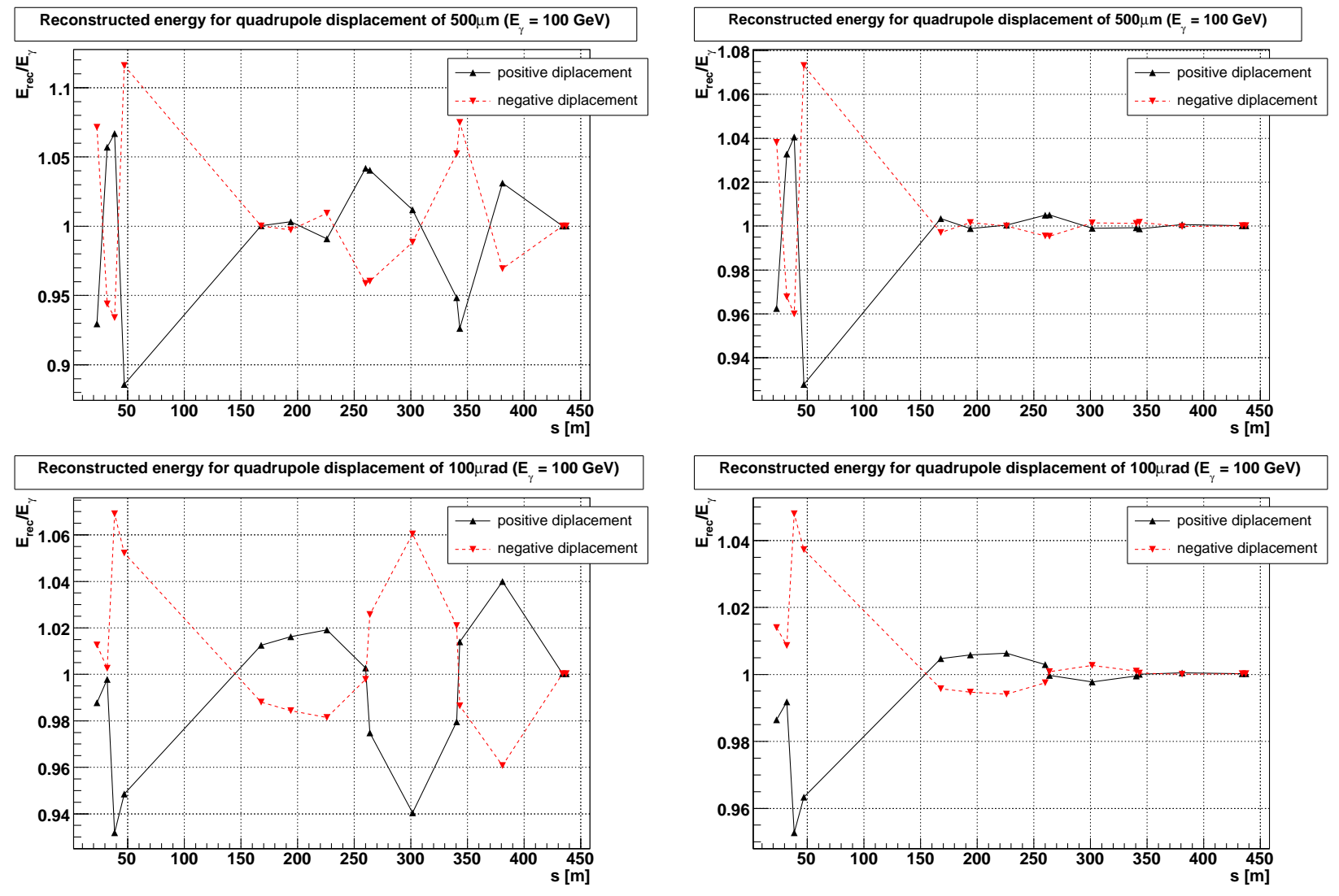

Figure 19: The misalignment of the LHC quadrupoles bias the energy reconstruction. The graphs show the bias for the reconstruction (with the trivial method) of a $100 \mathrm{GeV}$ energy loss assuming the misaligned quadrupoles at various positions. Each element is separately shifted $(500 \mu \mathrm{m})$ or tilted(100 $\mu \mathrm{rad})$, assuming a perfect alignment for the rest of the beamline. The impact of the misalignment can be important. Even a perfect knowledge of the actual beam position at the VFD (right) does not compensate for this bias, depending on the position of the misplaced quadrupole.

\section{Summary and outlook}

A new tool for the transport of particles in beamlines was presented, exhibiting high performances thanks to the transfer matrix model. The tool was validated with the MAD-X software, on the LHC beamlines. Much information is produced from the raw transport of particles and study of the transport up to forward detectors is subsequently possible. The reconstruction of variables at IP is tested as well as the impact of the misalignment of beamline optical elements on this reconstruction.

Hector is being ported in the software of the CMS experiment. Finally, further studies on the reconstruction methods, effects of the beamline misalignments and the related corrections will be led.

\section{Acknowledgments}

The authors are grateful to Monika Grothe, Pierre Van Mechelen, Valentina Avati and Samim Erhan for the useful discussions and comments. 


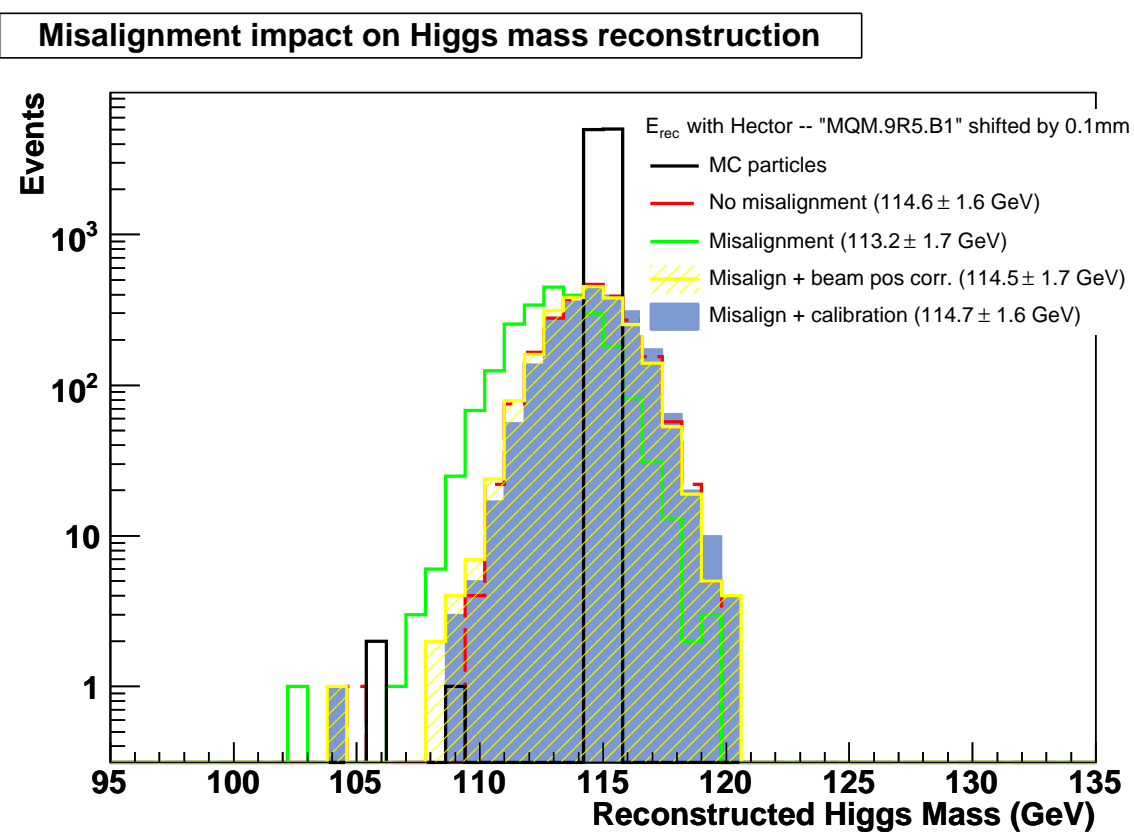

\section{Misalignment impact on Higgs mass reconstruction}

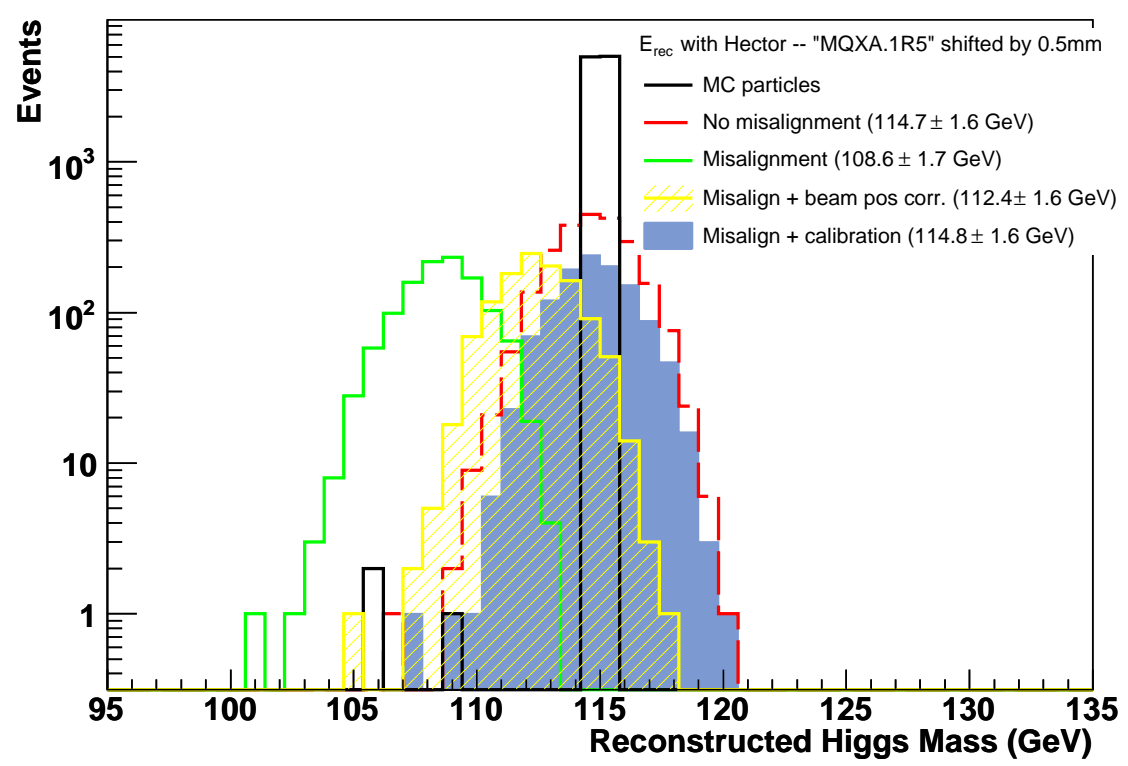

Figure 20: Illustration of the effects in the energy reconstruction due to the misalignment of LHC quadrupoles. The graphs show the reconstructed Higgs boson mass in the two-photon exclusive production, using energy of two forward scattered protons. In the upper plot, a quadrupole (MQM9R5, $s=347 \mathrm{~m}$ ) close to the detector has been shifted by $100 \mu \mathrm{m}$. Misaligning an optical element (MQXA1R5, $s=29 \mathrm{~m}$ ) close to the IP leads to a loss of acceptance (lower plot). The reconstructed values including the correction due to the dimuon calibration is also plotted. In brackets, the average reconstructed mass and its resolution are given, without including the beam energy dispersion. 


\section{Appendix}

\section{A Transfer matrices}

We define the transport matrices of optical elements as the following ones. The length of the optical element is $l$ and $k$ represents its magnetic field. We added the 6 th line and row in order to take the kickers into account. The energy dependence of the magnetic deflection of the particle is set by rescaling the $k$ factor by $\frac{E}{E-\Delta E}$. Note : Our convention is transposed compared to [5]: $x_{0} \cdot M=x_{1}$ instead of $x_{1}=M \cdot x_{0}$ so the matrices should be transposed. The matrices should have the following units :

$$
\mathbf{M}_{\text {units }}=\left(\begin{array}{cccccc}
1 & 1 / m & 1 & 1 / m & \mathrm{GeV} / m & 1 \\
m & 1 & m & 1 & \mathrm{GeV} & 1 \\
1 & 1 / m & 1 & 1 / m & \mathrm{GeV} / \mathrm{m} & 1 \\
m & 1 & m & 1 & \mathrm{GeV} & 1 \\
m / G e V & 1 / G e V & m / G e V & 1 / G e V & 1 & 1 \\
1 & 1 & 1 & 1 & 1 & 1
\end{array}\right)
$$

For a vertically focusing quadrupole (H_VerticalQuadrupole), where $\omega(k)=l \sqrt{k}$ :

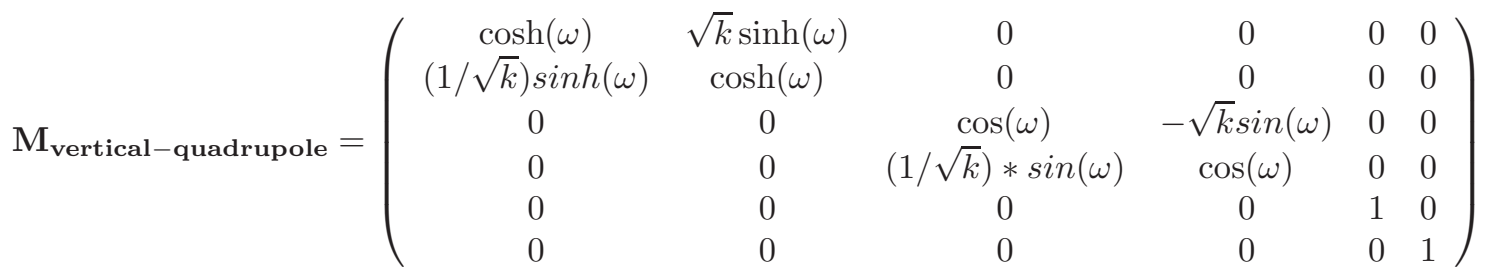

For a horizontally focusing quadrupole (H_HorizontalQuadrupole), where $\omega(k)=l \sqrt{k}$ :

$$
\mathbf{M}_{\text {horizontal-quadrupole }}=\left(\begin{array}{cccccc}
\cos (\omega) & -\sqrt{k} \sin (\omega) & 0 & 0 & 0 & 0 \\
(1 / \sqrt{k}) \sin (\omega) & \cos (\omega) & 0 & 0 & 0 & 0 \\
0 & 0 & \cosh (\omega) & \sqrt{k} \sinh (\omega) & 0 & 0 \\
0 & 0 & (1 / \sqrt{k}) \sinh (\omega) & \cosh (\omega) & 0 & 0 \\
0 & 0 & 0 & 0 & 1 & 0 \\
0 & 0 & 0 & 0 & 0 & 1
\end{array}\right)
$$

For a rectangle dipole (H_RectangularDipole), where $r(k)=1 / k$ :

$$
\mathbf{M}_{\text {rectangular-dipole }}=\left(\begin{array}{cccccc}
\cos (l / r) & \frac{-1}{r} \sin (l / r) & 0 & 0 & 0 & 0 \\
r \sin (l / r) & \cos (l / r) & 0 & 0 & 0 & 0 \\
0 & 0 & 1 & 0 & 0 & 0 \\
0 & 0 & l & 1 & 0 & 0 \\
2 r \sin ^{2}(l / 2 r) / B E & \sin (l / r) / B E & 0 & 0 & 1 & 0 \\
0 & 0 & 0 & 0 & 0 & 1
\end{array}\right)
$$

Note $: r *(1-\cos (l / r))$ causes a numerical sensitivity. We use $2 \sin ^{2}(x / 2)=1-\cos (x)$ instead. The rectangular shape implies an edge focusing of the dipole. In the beam frame, we apply the edge focusing as described in [5] :

$$
M_{\text {rdip-edge-focusing }}=M_{\text {edge }} M_{\text {rdip }} M_{\text {edge }}
$$


where $\Psi=l / 2 r$ and

$$
\mathbf{M}_{\text {edge }}=\left(\begin{array}{cccccc}
1 & \tan (\Psi) / r & 0 & 0 & 0 & 0 \\
0 & 1 & 0 & 0 & 0 & 0 \\
0 & 0 & 1 & -\tan (\Psi) / r & 0 & 0 \\
0 & 0 & 0 & 1 & 0 & 0 \\
0 & 0 & 0 & 0 & 1 & 0 \\
0 & 0 & 0 & 0 & 0 & 1
\end{array}\right)
$$

For a sector dipole (H_SectorDipole), either with their bending effect on or off, where $r(k)=1 / k$ :

$$
\begin{array}{r}
\mathbf{M}_{\text {sector-dip-bending-off }}=\left(\begin{array}{cccccc}
\cos (l / r) & \frac{-1}{r} \sin (l / r) & 0 & 0 & 0 & 0 \\
r \sin (l / r) & \cos (l / r) & 0 & 0 & 0 & 0 \\
0 & 0 & 1 & 0 & 0 & 0 \\
0 & 0 & l & 1 & 0 & 0 \\
0 & 0 & 0 & 0 & 1 & 0 \\
0 & 0 & 0 & 0 & 0 & 1
\end{array}\right) \\
\mathbf{M}_{\text {sector-dip-bending-on }}=\left(\begin{array}{cccccc}
\cos (l / r) & \frac{-1}{r} \sin (l / r) & 0 & 0 & 0 & 0 \\
r \sin (l / r) & \cos (l / r) & 0 & 0 & 0 & 0 \\
0 & 0 & 1 & 0 & 0 & 0 \\
0 & 0 & l & 1 & 0 & 0 \\
2 r \sin ^{2}(l / 2 r) / B E & \sin (l / r) / B E & 0 & 0 & 1 & 0 \\
0 & 0 & 0 & 0 & 0 & 1
\end{array}\right)
\end{array}
$$

The bending can be switched off when working in the detector frame. Obviously, this should not be used for non nominal energy beam particles.

For a drift (H_Drift) :

$$
\mathbf{M}_{\mathrm{drift}}=\left(\begin{array}{cccccc}
1 & 0 & 0 & 0 & 0 & 0 \\
l & 1 & 0 & 0 & 0 & 0 \\
0 & 0 & 1 & 0 & 0 & 0 \\
0 & 0 & l & 1 & 0 & 0 \\
0 & 0 & 0 & 0 & 1 & 0 \\
0 & 0 & 0 & 0 & 0 & 1
\end{array}\right)
$$

For a horizontal kicker (H_HorizontalKicker), where $k$ is the angular kick in rad :

$$
\mathbf{M}_{\text {horizontal-kicker }}=\left(\begin{array}{cccccc}
1 & 0 & 0 & 0 & 0 & 0 \\
l & 1 & 0 & 0 & 0 & 0 \\
0 & 0 & 1 & 0 & 0 & 0 \\
0 & 0 & l & 1 & 0 & 0 \\
0 & 0 & 0 & 0 & 1 & 0 \\
l \tan (k) / 2 & k & 0 & 0 & 0 & 1
\end{array}\right)
$$

For a vertical kicker (H_VerticalKicker), where $k$ is the angular kick in rad :

$$
\mathbf{M}_{\text {vertical-kicker }}=\left(\begin{array}{cccccc}
1 & 0 & 0 & 0 & 0 & 0 \\
l & 1 & 0 & 0 & 0 & 0 \\
0 & 0 & 1 & 0 & 0 & 0 \\
0 & 0 & l & 1 & 0 & 0 \\
0 & 0 & 0 & 0 & 1 & 0 \\
0 & 0 & l \tan (k) / 2 & k & 0 & 1
\end{array}\right)
$$




\section{B User guide}

\section{B.1 The usual "getting started" chapter}

In order to run HECTOR on your system, first download its sources and compile the libHector library ${ }^{3}$ :

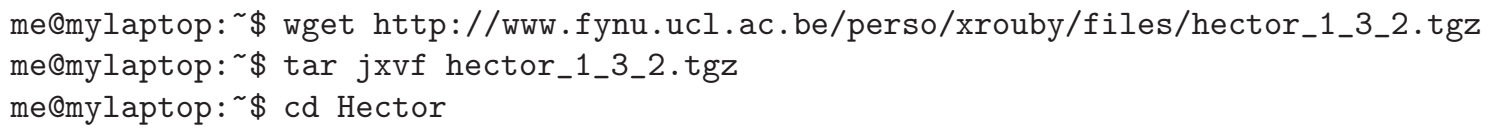

Hector has been tested with Root versions from 4.xx. When launched from Hector's main directory, Root will automatically load libHector (see rootlogon.C) and display a welcome prompt. In

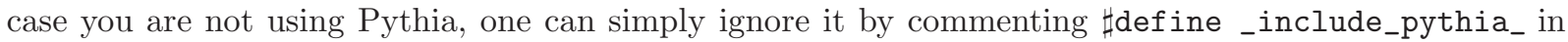
./include/H_Parameters.h ${ }^{4}$.

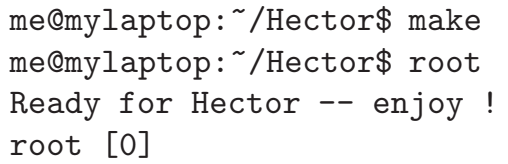

As a general comment, don't forget that every class you use in your routine should be included in the routine file, for instance :

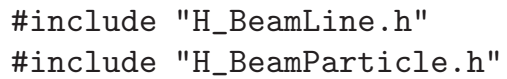

\section{B.2 The everyday life with Hector}

In this chapter, we will tell you how to use HECTOR to perform basics tasks such as using predefined beamlines and designing yours, propagating particles along those, and computing some beam properties. Generally speaking, the detailed constructors and methods arguments for HECTOR classes will not be detailed here, as it can be easily found in the Internet reference manual ${ }^{5}$.

\section{B.2.1 Genesis 1 : the beamline}

The first step to use HECTOR is to define the list of optical elements that the particles will cross while in the beam pipe. This can be done in two ways :

- Using (existing) magnets tables

- Building the line yourself

\section{Tables of optical elements}

This way is the simplest. You only need a text file containing columns with the magnet name, position, length, strength. Apertures can also be specified in this file. The order of the columns is meaningless but you should use column headers with the following "codenames" :

\footnotetext{
${ }^{3}$ The Makefile is compatible for a compilation on Linux and Windows/Cygwin systems. For such Windows, just modify the library extension name to $\mathrm{LEXT}=$ dll in the Makefile.

${ }^{4}$ If your Roот version does not contain the PyтніA libraries, the make command will return an error. In that case, you will have to remove by hand the references to libPythia and libEGPythia in the Makefile, then run "make clean" and "make".

${ }^{5}$ This online manual, as well as all information on Hector, can be found here : http://www.fynu.ucl.ac.be/hector.html
} 
- NAME : name

- KEYWORD : type

- $\mathrm{S}$ : position along the line

- L : length

- K0L : dipole strength (horizontal)

- K1L : quadrupole strength

- HKICK : horizontal kick angle

- VKICK : vertical kick angle

- APERTYPE : aperture type

- APER_X : aperture size (X runs from 1 to 4 )

The "KEYWORD" code defining the element type can take the following values :

- "DRIFT" : no-field zone ${ }^{6}$

- "QUADRUPOLE" : regular quadrupole

- "RBEND" : rectangular dipole

- "SBEND" : sector dipole

- "VKICKER" : vertical kicker

- "HKICKER" : horizontal kicker

- "MARKER" : dummy element ${ }^{7}$

- "RCOLLIMATOR" : rectangular collimator

The "APERTYPE" can be :

- "NONE" : no aperture limitation

- "CIRCLE" : circular aperture. the radius is given by APER_1.

- "RECTANGLE" : rectangular aperture. The $x$ and $y$ sizes are given by APER_1 and APER_2.

- "ELLIPSE" : elliptic aperture. Same size definition as the rectangular aperture.

- "RECTELLIPSE" : intersection between a rectangle and an ellipse. The APER_X parameters give the rectangle then the ellipse size.

When your table is properly written, the method to create your beamline is the following one ${ }^{8}$ :

\footnotetext{
${ }^{6}$ The drift spaces lines in the tables are not read by НестоR, because it automatically fills the gaps between other elements with drifts of the right length.

${ }^{7}$ This can be used to tag special places such as Interaction points.

${ }^{8}$ As all the methods/functions using a H_BeamLine as argument require it to have the "pointer" type, it is heavily recommended to declare it with that type in all the cases.
} 


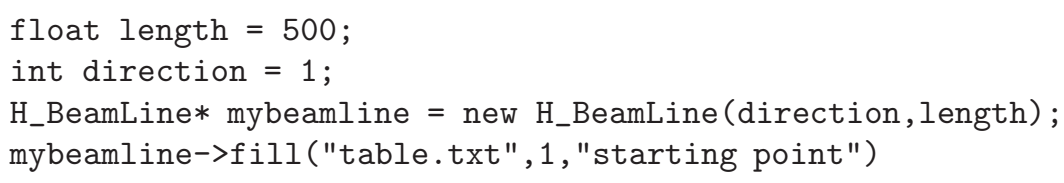

The two arguments of the $H_{\_}$BeamLine creator are the line length and the direction in which the table will be read (forwards (1) or backwards (-1)). The fill Method requires the name of the table file, the propagation direction of the particles from the starting point and the name of the starting point marker.

The meaning of these two direction parameters can seem unclear, so let's suppose you use the following table :

$\begin{array}{llrl}\text { NAME } & \text { KEYWORD } & \text { S } & \ldots \\ \text { "element1" } & \text { "rbend" } & 0 & \ldots \\ \text { "element2" } & \text { "vquadrupole" } & 10 & \ldots \\ \text { "starting point" } & \text { "marker" } & 20 & \ldots \\ \text { "element3" } & \text { "vquadrupole" } & 30 & \ldots \\ \text { "element4" } & \text { "rbend" } & 40 & \ldots\end{array}$

If the $H_{-}$BeamLine creator argument is 1 , we read only the last three elements. Has it been -1 , we would have read the first three. The fill argument being 1, the particles will go from top to bottom. If it is -1 , it goes from bottom to top ${ }^{9}$.

Examples of elements tables - taken from the LHC IP5 region - can be found in the "data" directory of HECTOR as a working example.

\section{Element-by-element beam building}

Another way to build the beamline is to add each element separately in the beamline. This is done by this kind of code :

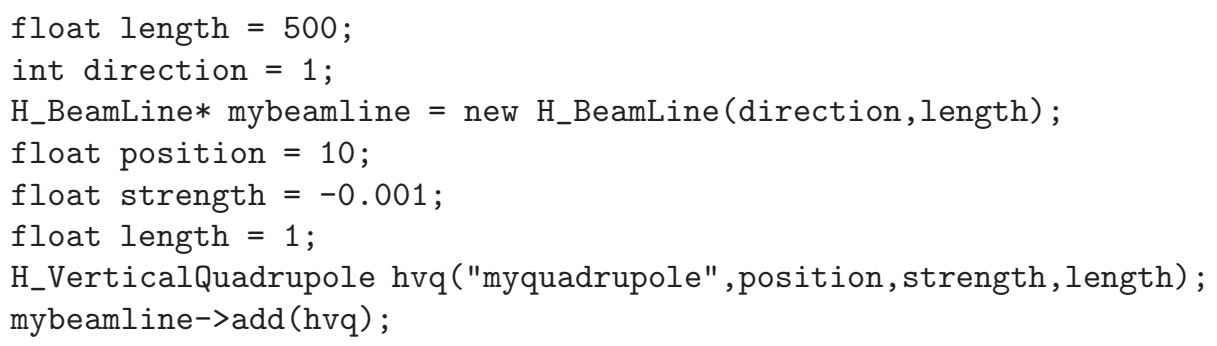

This way is not recommended, as the other one is more convenient in most of the cases. But it still can be used to add markers or user-defined elements useful only in specific routines, or to design a new beamline automatically from Monte Carlo techniques.

It is important to note that the $H_{\_}$Dipole and $H_{-} Q u a d r u p o l e$ classes are purely abstract and should then never be used directly.

\section{Alignment effects}

After building the beamline, one can move elements around their nominal position using the following method :

\footnotetext{
${ }^{9}$ It can be useful to notice that the order of the lines does not matter as the ordering is done from the $\mathrm{S}$ parameter. However, the elements have to be on their real position (before or after) with respect to the starting point.
} 


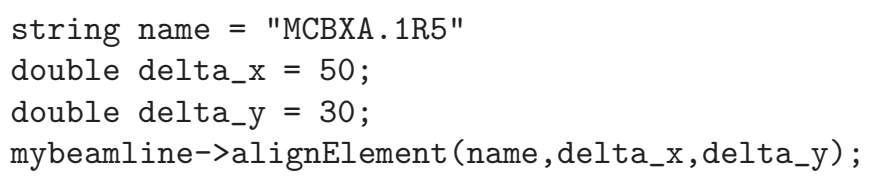

This will displace the element called $M C B X A \cdot 1 R 5^{10}$ by the specified amounts in the $\mathrm{x}$ and y directions. Corresponding angles can be changed using the similar method :

mybeamline->alignElement (name, delta_theta_x, delta_theta_y);

\section{B.2.2 Genesis 2 : The particles}

Using the lines :

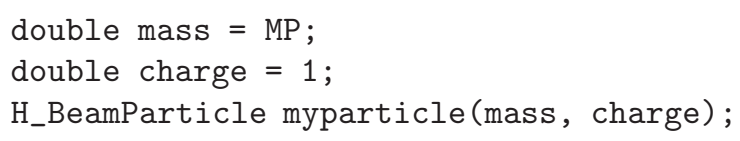

one particle with given mass and charge ${ }^{11}$ is created at the "starting point" (IP) with energy defined by the BE (beam energy) given in the $H_{-}$Parameters.h file. The other main variables in this file are :

- $\mathrm{SBE}$ : beam energy dispersion

- PX : $x$ position at IP

- PY : y position at IP

- TX : $x$ angle at IP

- TY : $y$ angle at IP

- SX : $x$ dispersion of the beam at IP

- SY : $y$ dispersion of the beam at IP

- STX : $x$ angle dispersion of the beam at IP

- STY : $y$ angle dispersion of the beam at IP

The $x$ and $y$ positions are always given in $\mu \mathrm{m}$ and the corresponding angles are in $\mu \mathrm{rad}$. The $s$ coordinate is conveniently expressed in meters.

The particle can then be smeared ${ }^{12}$ using the SBE, SX, SY, STX, STY parameters by the following methods :

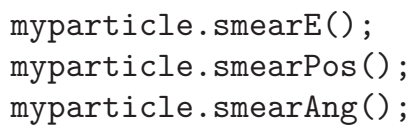

Other useful methods for the particles at the starting point include :

H_BeamParticle: :emitGamma(float Energy, float virtuality);

\footnotetext{
${ }^{10}$ Please note that in LHC tables the names include the double quotes, meaning that the name should be something like "’MCBXA.1R5\"'.

${ }^{11}$ If not specified, the default particle used is a proton.

${ }^{12}$ These methods use a simple Gaussian smearing.
} 
which allows to simulate the emission of a virtual particle with given properties, causing an energy loss and an angle displacement. Another way to simulate an energy change is to use the

$$
\text { H_BeamParticle: : } \operatorname{setE(float~Energy);~}
$$

method which only changes the energy parameter of the particle without any other effects. The effect of the energy loss is obviously simulated by HECTOR ${ }^{13}$.

\section{B.2.3 Exodus : the particle propagation}

Once the beamline has been set and the particle has all the desired properties, the latter can be propagated along the former using the following :

myparticle.computePath (mybeamline);

This computes the positions and angles of the particle at the entrance of each element and allows to interpolate its position in all the drift spaces. This method is the most time-consuming in HECTOR and thus defines the speed of the program. The typical timescale is less than $4 \mu$ s per particle and per element (including drifts) $)^{14}$.

\section{Aperture check}

If one wants to check if the particle has been stopped by any aperture, the method following

bool stopped = myparticle.stopped (mybeamline);

should be used. It checks all the element apertures and stores the closest intercepting element from the IP. This element can then be obtained using :

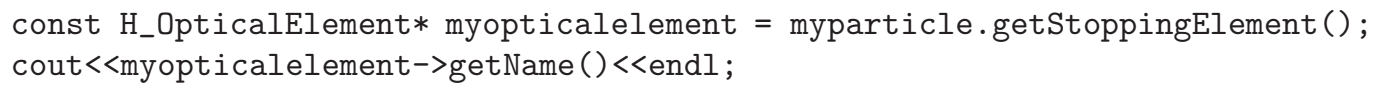

for instance to show the name of the element.

\section{Getting the particle information}

Once the path has been computed, it is quite legitimate to be interested on the particle transverse position $^{15}$ and angle at some position in s. This is achieved the following methods :

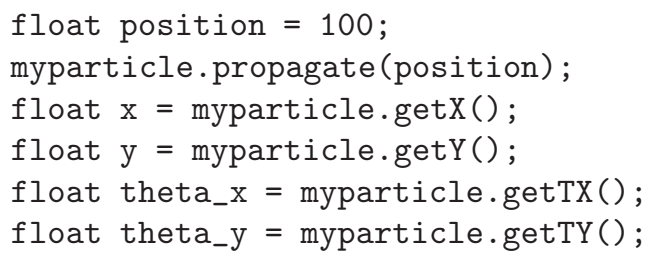

The particle can then be "propagated" to any other other position and the variables obtained at that new positions by the same methods. The complete path can be obtained via the Root TGraph class using the following :

\footnotetext{
${ }^{13}$ The energies and virtualities are always given in $\mathrm{GeV}$ and $\mathrm{GeV}^{2}$. The virtuality is by definition a negative number.

${ }^{14}$ Tested on a $1.7 \mathrm{GHz}$ Centrino processor running Linux.

${ }^{15}$ The $x$ and $y$ coordinates are respectively the transverse horizontal and vertical displacement of the particle.
} 


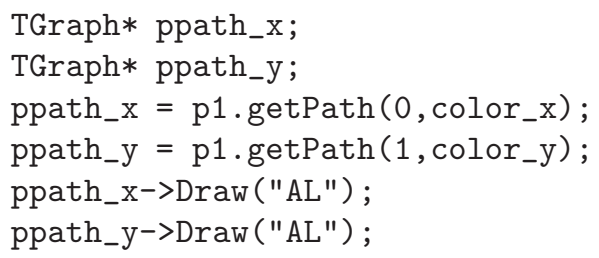

A convenient way to deal with big numbers of particles is to use the H_Beam class. It builds the beam in the following way :

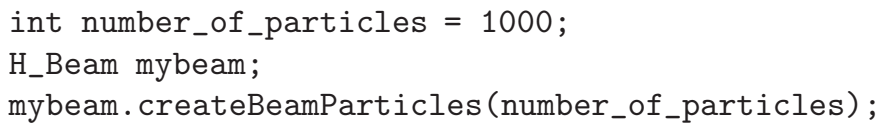

where the createBeamParticles method smears all the particle variables using the pre-defined parameters. Most of the single-particle methods can then be applied to the full beam, such as computePath and propagate. One can access one particle of the beam using the simple

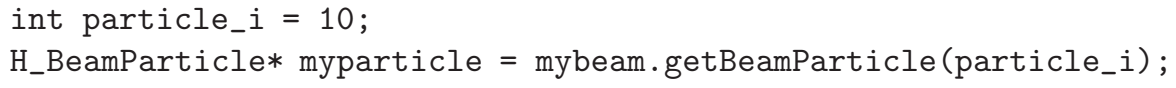

for instance to get it's properties or to use methods such as emitGamma on it. Using a $H \_B e a m$ is especially useful when the user is interested in global properties of the beam, such as its spatial extension, given by the $\beta$ functions. This is achieved in a straightforward way using the method :

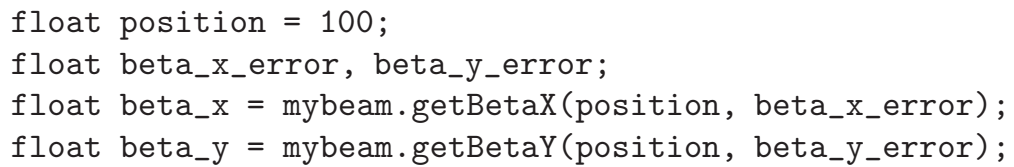

which returns the value of the $\beta$ function at the given position and the error on this value.

\section{B.2.4 Kings : Remarks for the courageous user}

In this section we will detail some features of HECTOR that most people should not use, some problems that will appear to only few of the users, and other exceptionally boring things that could help you in some very seldom cases.

\section{Absolute frame}

First of all, HECTOR is designed to work in "relative frame". This means the normal transverse position for a particle with no angle or displacement at the IP is always 0, and then particles with nominal beam energy will not be deflected by the dipoles.

However, in some case it can be interesting to check the absolute trajectory of the beam. This can be achieved with HECTOR, if and only if you can consider all your beam elements as parallel. In that case, it suffices to add two lines at the beginning of your routine :

extern bool relative_energy;

relative_energy = false;

You can also displace all beam elements after a given point laterally using the following : 


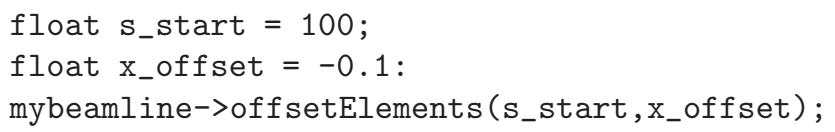

This will displace horizontally all elements from $100 \mathrm{~m}$ onwards from the IP, by $10 \mathrm{~cm}$ in the negative $x$ direction.

\section{Kickers}

One exception to the "no-deflection" rule of the previous section is obviously the presence of an initial angle of the beam compared to its natural propagation direction, for instance a crossing angle. This kind of case is usually coped with using "kickers" which deflect the beam by a given angle. These kickers are obviously included in НестоR and are the only elements that doesn't respect the "no-deflection" law. If one feels more comfortable to switch this effect off, it is easily be done by including :

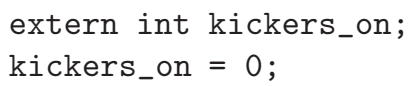

at the beginning of the routine.

\section{B.2.5 Revelation : compiling and running your code}

Once you have written a routine and made sure you put it in the right place - for instance the very convenient HECTOR/routines directory - you only need to compile it. This is most easily done by running RoOT and doing the following ${ }^{16}$ :

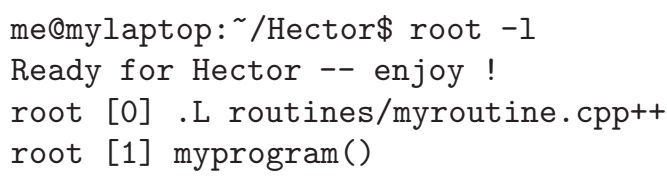

\section{B.3 A simple example}

As a reward for your patience, here's a little working example of HeCTOR plotting a transverse view of the beam at $220 \mathrm{~m}$ from the IP.

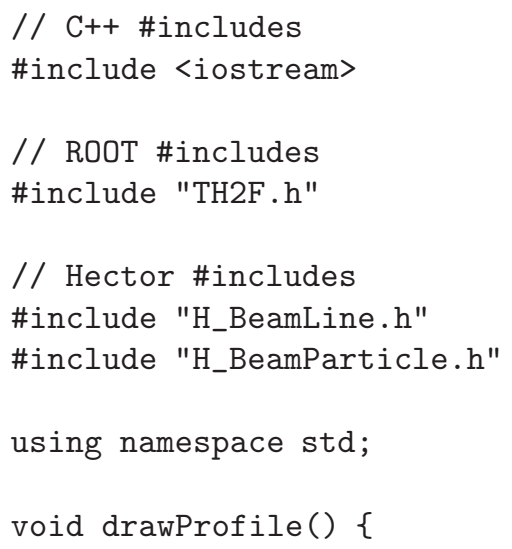

\footnotetext{
${ }^{16}$ Here we suppose that HECTOR is already properly installed on your computer.
} 


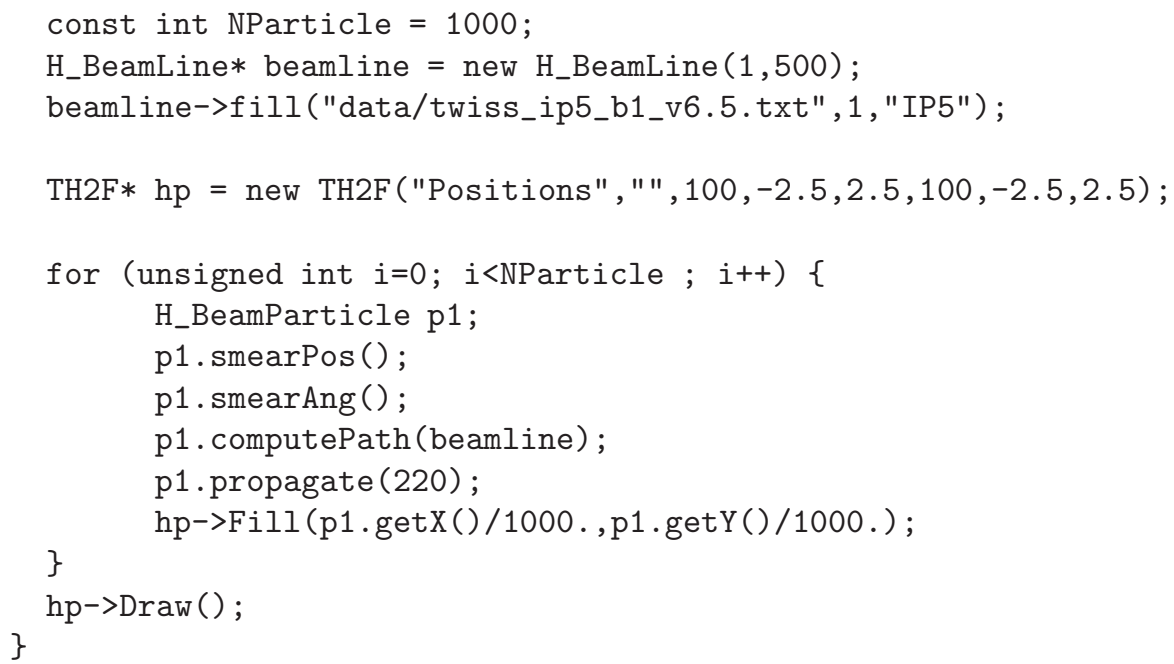

\section{References}

[1] Root - An Object Oriented Data Analysis Framework, R. Brun and F. Rademakers, Nucl. Inst. \& Meth. in Phys. Res. A 389 (1997) 81-86, http://root.cern.ch

[2] MAD, CERN-SL-2000-026, F.C. Iselin, J.M. Jowett, J. Pancin, A. Adelmann

[3] EDMS http://lhc.web.cern.ch/lhc/

[4] LHC Design report volume I, CERN-2004-003 Geneve (2004), O.S. Brüning, P. Collier, P. Lebrun, S. Myers, R. Ostojic, J. Poole, P. Proudlock, http://ab-div.web.cern.ch/ab-div/Publications/LHC-DesignReport.html

[5] The Physics of Particle Accelerators, K. Wille, Oxford (2006), ISBN 019850549

[6] Prospects for Diffractive and Forward Physics at the LHC, The CMS and TOTEM diffractive and forward physics working group, CERN/LHCC 2006-039/G-124

[7] K. Piotrzkowski, Tagging two-photon production at the CERN Large Hadron Collider, Phys. Rev D63 (2001) 071502

[8] PYTHia event generator, T. Sjöstrand, L. Lönnblad, S. Mrenna, hep-ph/0603175

[9] LHC optics http://proj-lhc-optics-web.web.cern.ch/

[10] Transport: a computer program for designing charged particle beam transport systems, K.L. Brown, F. Rothacker, D.C. Carey, F.C. Iselin, CERN 80-04 


\section{Contents}

1 Introduction $\quad 1$

2 The LHC beamlines $\quad 2$

2.1 Beamline elements and beam parameters. . . . . . . . . . . . . . . . . 2

2.2 Beamline map . . . . . . . . . . . . . . . . . . . . . . . . 3

3 Simulation techniques $\quad 3$

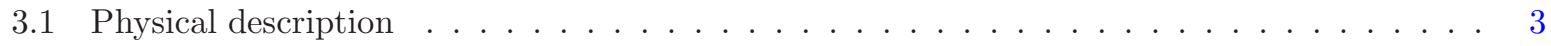

3.2 Implementation . . . . . . . . . . . . . . . . . . . . . . . . . . 4

4 Validation and performances $\quad \mathbf{5}$

4.1 Performances . . . . . . . . . . . . . . . . . . . . . . . . . 5

4.2 Validating Hector with MAD-X . . . . . . . . . . . . . . . . . . . 5

4.3 Validating off-beam energy simulation . . . . . . . . . . . . . . . . . . 6

5 Beamline simulation $\quad 9$

5.1 Trajectories . . . . . . . . . . . . . . . . . . . . . . 9

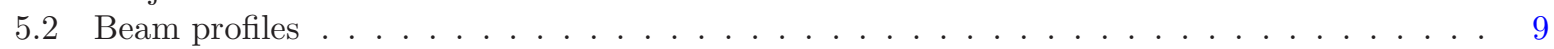

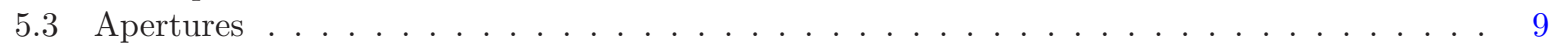

6 Very Forward Detectors $\quad 14$

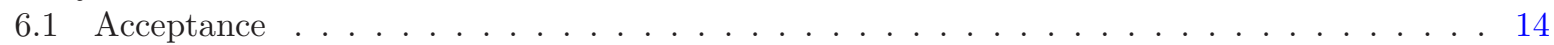

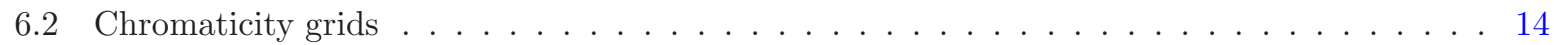

6.3 Irradiation levels . . . . . . . . . . . . . . . . . . . . . . . . . . 14

6.4 Reconstruction . . . . . . . . . . . . . . . . . . . . . . . . . . 19

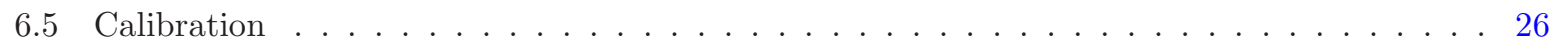

6.6 Misalignments . . . . . . . . . . . . . . . . . . . . . . . . 26

$\begin{array}{lll}7 & \text { Summary and outlook } & 27\end{array}$

$\begin{array}{lr}\text { A Transfer matrices } & 29\end{array}$

B User guide $\quad 31$

B.1 The usual "getting started" chapter . . . . . . . . . . . . . . . . . . . . . . . 31

B.2 The everyday life with Hector . . . . . . . . . . . . . . . . . . . . . . . . 31

B.2.1 Genesis 1 : the beamline . . . . . . . . . . . . . . . . . 31

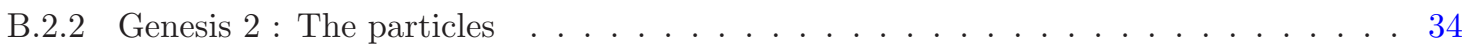

B.2.3 Exodus : the particle propagation . . . . . . . . . . . . . . . . . 35

B.2.4 Kings : Remarks for the courageous user . . . . . . . . . . . . . . . 36

B.2.5 Revelation : compiling and running your code . . . . . . . . . . . . . . 37

B.3 A simple example . . . . . . . . . . . . . . . . . . . . . . 37 


\section{List of Figures}

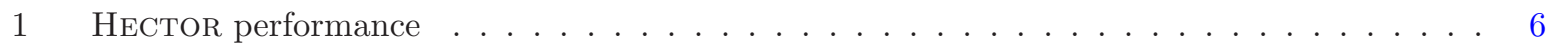

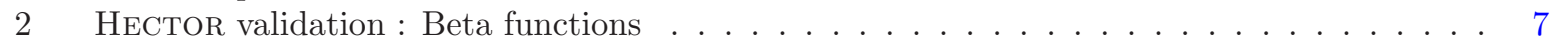

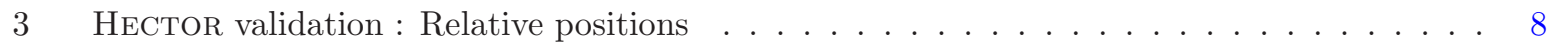

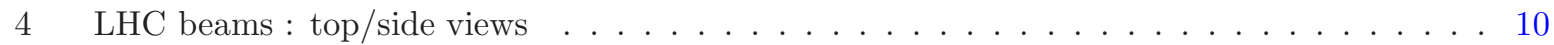

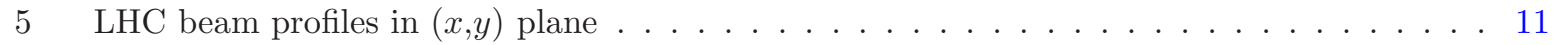

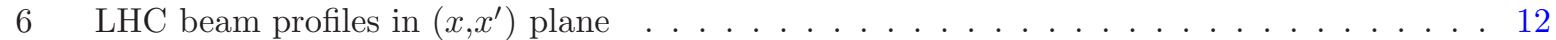

7 Example of aperture check . . . . . . . . . . . . . . . . . . . . . 13

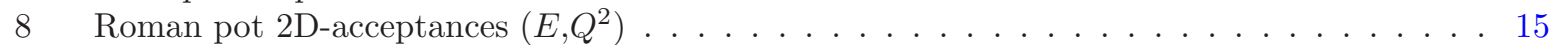

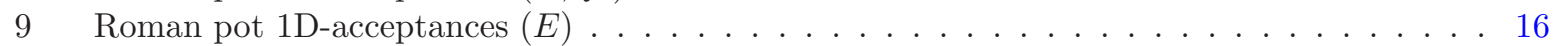

10 Chromaticity grids: Dependence on energy and virtuality loss at the IP $\ldots \ldots \ldots$. . . . 17

11 Dependence on vertical angle at IP . . . . . . . . . . . . . . . . . . 17

12 Irradiation levels from $\mathrm{pp} \rightarrow \mathrm{pX} \ldots \ldots \ldots \ldots \ldots \ldots$

13 Resolution of the reconstructed energy . . . . . . . . . . . . . . . . . . . . . 21

14 Bias studies for the energy reconstruction . . . . . . . . . . . . . . . . . 22

15 Reconstruction of the hor angle at IP . . . . . . . . . . . . . . . . . . . . 23

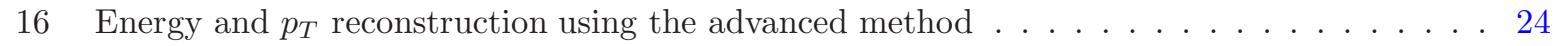

17 Reconstructed $p_{T}$ resolution using the advanced method . . . . . . . . . . . . 25

18 Reconstructed Energy resolution using the advanced method . . . . . . . . . . . . . 25

19 Reconstruction error due to the misalignment of quadrupoles . . . . . . . . . . . . . 27

20 Higgs two-photon exclusive production: error on reconstructed mass from misalignment . . 28 